\title{
On a parabolic quadrilateral finite element for compressible flows
}

\author{
Dena Hendriana, Klaus Jürgen Bathe * \\ Department of Mechanical Engineering, Massachusetts Institute of Technology, 77 Massachusetts Avenue, Cambridge, MA 02139, USA
}

Dedicated to Prof. J.T. Oden on the occasion of his 60th birthday

\begin{abstract}
We present a 9-node finite element for compressible flow solutions. A high-order derivative upwind term and a shock capturing term are employed for stability and accuracy of the formulation. We give the solutions of various example problems to illustrate our experiences with the element. (C) 2000 Published by Elsevier Science S.A. All rights reserved.
\end{abstract}

\section{Introduction}

The numerical solution of the governing equations of high-speed compressible flows entails many difficulties due to the presence of shocks, viscous boundary layers and their interactions in the domain of interest. To solve compressible flow problems using the usual low-order control volume and finite difference procedures, extremely fine meshes are used, in particular, in the areas where boundary layers and their interactions with shocks occur, to obtain reasonably accurate results. Similar fine meshes are also required when using the usually employed low-order finite element techniques, although of course the use of unstructured meshes allows the use of coarser meshes in some regions of the flow. The reason for requiring the fine meshes are the low-order convergence behavior of the finite elements, both for the diffusive and the convective terms in the Navier-Stokes equations. This observation motivates us to study the possibility of developing a parabolic quadrilateral element for compressible flows. The element would naturally provide a higher convergence behavior for the diffusive terms, and - provided effective upwinding and shock capturing schemes are embedded in the element - should also give a higher accuracy for the convective terms of the flow equations [1].

Many methods have been proposed to obtain numerical solutions of compressible flow problems using a linear (that is, low-order) triangular element [2-7] or using a linear quadrilateral element [8-10]. Also, Shapiro endeavored to develop a parabolic quadrilateral element but the author did not entirely use parabolic functions as the convective term was discretized using linear interpolations [9]. Oden et al. developed $h-p$ discretization schemes that include, of course, higher-order elements [11-14]. These schemes are based upon the Taylor-Galerkin method, which has second order accuracy in time, to discretize the time and space variables, and to stabilize the convective term, an artificial diffusion is employed.

Oscillations might occur in the numerical solutions at the locations where the convective term is dominant and to eliminate these oscillations, an upwind method need be used. The SUPG method, proposed by Hughes et al. originally for incompressible flows [15], is a very popular upwind technique used in finite

\footnotetext{
${ }^{*}$ Corresponding author. Tel.: +1-617-253-6645; fax: +1-617-253-2275.
} 
element discretizations. The use of the SUPG technique and modifications thereof for compressible flow problems have been proposed in several contributions $[5,8,16]$ but only linear elements were used. Some authors extended the SUPG technique also for use with a quadratic element, but only the scalar, convective-diffusive problem and not the system of Navier-Stokes equations was considered [17-20].

In the present paper, we propose a new upwind method for a parabolic quadrilateral element to solve high-speed compressible flows. The upwinding is similar to what is used in the finite difference method.

In a quadratic element, the nodal connectivity is wider than in a linear element. This nodal connectivity makes it possible to design a higher order upwind method [21]. The upwind method that we propose contains second order derivatives, hence the numerical results using this upwind technique are expected to have a higher order accuracy.

In the presence of a shock, a stable numerical method still gives solutions with oscillations around the shock and to reduce these oscillations, a shock capturing method is needed. We propose here a shock capturing procedure for the quadratic element compatible in error with the accuracy obtained using the new upwind term.

In the next sections we first present the finite element formulation of the 9-node element, and then we present the solutions of various low and high Mach number problems obtained with the element.

\section{Governing equations}

The fluid flow is modeled with the Navier-Stokes equations that contain the conditions of conservation of mass, momentum and energy. In vector form, the Navier-Stokes equations can be written as

$$
\boldsymbol{U}_{, t}+\boldsymbol{F}_{j, j}-\boldsymbol{G}_{j, j}-\boldsymbol{R}=\mathbf{0},
$$

where ()$_{, t}$ and ()$_{, j}$ denote derivatives with respect to time and the $x_{j}$-coordinate. We consider only the twodimensional case $(i, j, k=1,2)$ for which

$$
\begin{aligned}
& \boldsymbol{U}=\left[\begin{array}{c}
\rho \\
\rho v_{1} \\
\rho v_{2} \\
\rho E
\end{array}\right], \\
& \boldsymbol{F}_{j}=\left[\begin{array}{c}
\rho v_{j} \\
\rho v_{1} v_{j}+(\gamma-1) \rho\left(E-\frac{1}{2} v_{k}^{2}\right) \delta_{1 j} \\
\rho v_{2} v_{j}+(\gamma-1) \rho\left(E-\frac{1}{2} v_{k}^{2}\right) \delta_{2 j} \\
\rho E v_{j}+(\gamma-1) \rho v_{j}\left(E-\frac{1}{2} v_{k}^{2}\right)
\end{array}\right], \\
& \boldsymbol{G}_{j}=\left[\begin{array}{c}
0 \\
\lambda v_{k, k} \delta_{1 j}+\mu\left(v_{1, j}+v_{j, 1}\right) \\
\lambda v_{k, k} \delta_{2 j}+\mu\left(v_{2, j}+v_{j, 2}\right) \\
\lambda v_{j} v_{k, k}+\mu v_{i}\left(v_{i, j}+v_{j, i}\right)+\frac{k}{c_{v}}\left(E-\frac{1}{2} v_{k}^{2}\right)_{, j}
\end{array}\right], \\
& \boldsymbol{R}=\left[\begin{array}{c}
0 \\
\rho f_{1}^{\mathrm{B}} \\
\rho f_{2}^{\mathrm{B}} \\
\rho v_{i} f_{i}^{\mathrm{B}}+\rho q^{\mathrm{B}}
\end{array}\right]
\end{aligned}
$$

where $\rho, v_{i}, E, f_{i}^{\mathrm{B}}, q^{\mathrm{B}}$ are density, velocity component in the $x_{i}$-direction, specific energy, body force in the $x_{i}$ - direction and body heat generation, respectively; and $\gamma, \mu, \lambda, k, c_{v}$ are the ratio of the specific heats, fluid viscosity, second viscosity coefficient, coefficient of thermal conductivity and the specific heat at constant volume, respectively; $\delta_{i j}$ is the Kronecker delta (i.e. $\delta_{i j}=1$ for $i=j$, and $\delta_{i j}=0$ for $i \neq j$ ). Here, we have used the constitutive relations for an ideal gas, the Newtonian stress-strain relation and Fourier's law of 
heat conduction. Taking the quasi-linear form of the convective term and rewriting the diffusive and source terms, the Navier-Stokes equations can be written as [23]

$$
\boldsymbol{U}_{, t}+\boldsymbol{A}_{j} \boldsymbol{U}_{, j}-\left(\boldsymbol{K}_{j i} \boldsymbol{U}_{, i}\right)_{, j}-\boldsymbol{S} \boldsymbol{U}=\mathbf{0},
$$

where the Jacobian matrices of $\boldsymbol{F}_{j}$ are

$$
\begin{aligned}
& \boldsymbol{A}_{1}=\left[\begin{array}{cccc}
0 & 1 & 0 & 0 \\
\frac{(\gamma-3)}{2} v_{1}^{2}+\frac{(\gamma-1)}{2} v_{2}^{2} & (3-\gamma) v_{1} & (1-\gamma) v_{2} & (\gamma-1) \\
-v_{1} v_{2} & v_{2} & v_{1} & 0 \\
-\gamma v_{1} E+(\gamma-1) v_{1}\left(v_{1}^{2}+v_{2}^{2}\right) & \gamma E-\frac{(\gamma-1)}{2}\left(3 v_{1}^{2}+v_{2}^{2}\right) & (1-\gamma) v_{1} v_{2} & \gamma v_{1}
\end{array}\right], \\
& \boldsymbol{A}_{2}=\left[\begin{array}{cccc}
0 & 0 & 1 & 0 \\
-v_{1} v_{2} & v_{2} & v_{1} & 0 \\
\frac{(\gamma-1)}{2} v_{1}^{2}+\frac{(\gamma-3)}{2} v_{2}^{2} & (1-\gamma) v_{1} & (3-\gamma) v_{2} & (\gamma-1) \\
-\gamma v_{2} E+(\gamma-1) v_{2}\left(v_{1}^{2}+v_{2}^{2}\right) & (1-\gamma) v_{1} v_{2} & \gamma E-\frac{(\gamma-1)}{2}\left(v_{1}^{2}+3 v_{2}^{2}\right) & \gamma v_{2}
\end{array}\right] .
\end{aligned}
$$

The matrices of the diffusive terms are

$$
\begin{aligned}
& \boldsymbol{K}_{11}=\left[\begin{array}{cccc}
0 & 0 & 0 & 0 \\
-\frac{\lambda v_{1}}{\rho}-\frac{2 \mu v_{1}}{\rho} & \frac{\lambda}{\rho}+\frac{2 \mu}{\rho} & 0 & 0 \\
-\frac{\mu v_{2}}{\rho} & 0 & \frac{\mu}{\rho} & 0 \\
c_{1} & \frac{\lambda v_{1}}{\rho}+\frac{2 \mu v_{1}}{\rho}-\frac{k v_{1}}{c_{v} \rho} & \frac{\mu v_{2}}{\rho}-\frac{k v_{2}}{c_{v} \rho} & \frac{k}{c_{v} \rho}
\end{array}\right], \\
& \boldsymbol{K}_{12}=\left[\begin{array}{cccc}
0 & 0 & 0 & 0 \\
-\frac{\lambda v_{2}}{\rho} & 0 & \frac{\lambda}{\rho} & 0 \\
-\frac{\mu v_{1}}{\rho} & \frac{\mu}{\rho} & 0 & 0 \\
-\frac{\lambda v_{1} v_{2}}{\rho}-\frac{\mu v_{1} v_{2}}{\rho} & \frac{\mu v_{2}}{\rho} & \frac{\lambda v_{1}}{\rho} & 0
\end{array}\right], \\
& \boldsymbol{K}_{21}=\left[\begin{array}{cccc}
0 & 0 & 0 & 0 \\
-\frac{\mu v_{2}}{\rho} & 0 & \frac{\mu}{\rho} & 0 \\
-\frac{\lambda v_{1}}{\rho} & \frac{\lambda}{\rho} & 0 & 0 \\
-\frac{\lambda v_{1} v_{2}}{\rho}-\frac{\mu v_{1} v_{2}}{\rho} & \frac{\lambda v_{2}}{\rho} & \frac{\mu v_{1}}{\rho} & 0
\end{array}\right], \\
& \boldsymbol{K}_{22}=\left[\begin{array}{cccc}
0 & 0 & & 0 \\
-\frac{\mu v_{1}}{\rho} & \frac{\mu}{\rho} & 0 & 0 \\
-\frac{2 v_{2}}{\rho}-\frac{2 \mu v_{2}}{\rho} & 0 & \frac{\lambda}{\rho}+\frac{2 \mu}{\rho} & 0 \\
c_{2} & \frac{\mu v_{1}}{\rho}-\frac{k v_{1}}{c_{v} \rho} & \frac{\lambda v_{2}}{\rho}+\frac{2 \mu v_{2}}{\rho}-\frac{k v_{2}}{c_{v} \rho} & \frac{k}{c_{v} \rho}
\end{array}\right],
\end{aligned}
$$

where

$$
\begin{aligned}
& c_{1}=-\frac{\lambda v_{1}^{2}}{\rho}-\frac{2 \mu v_{1}^{2}}{\rho}-\frac{\mu v_{2}^{2}}{\rho}-\frac{k E}{c_{v} \rho}+\frac{k v_{1}^{2}}{c_{v} \rho}+\frac{k v_{2}^{2}}{c_{v} \rho}, \\
& c_{2}=-\frac{\lambda v_{2}^{2}}{\rho}-\frac{\mu v_{1}^{2}}{\rho}-\frac{2 \mu v_{2}^{2}}{\rho}-\frac{k E}{c_{v} \rho}+\frac{k v_{1}^{2}}{c_{v} \rho}+\frac{k v_{2}^{2}}{c_{v} \rho}
\end{aligned}
$$


and the source matrix is

$$
\boldsymbol{S}=\left[\begin{array}{cccc}
0 & 0 & 0 & 0 \\
f_{1}^{B} & 0 & 0 & 0 \\
f_{2}^{B} & 0 & 0 & 0 \\
q^{B} & f_{1}^{B} & f_{2}^{B} & 0
\end{array}\right]
$$

The boundary conditions that we consider correspond to subsonic, transonic and supersonic flows; and appropriate flux boundary values are used to establish well-posed problems, see Ref. [23]. We impose the boundary conditions in terms of the solution variables, density, momentum and total energy. For the specification of other boundary values, we employ constraint equations.

\section{Finite element discretization}

In this section, we discuss the finite-dimensional spaces used for the quadratic element and give the finite element formulation. The artificial diffusion to stabilize the convective terms, and the shock capturing term to reduce oscillations around shocks and other discontinuities are presented.

\subsection{Finite element spaces}

Consider a finite element discretization of the fluid domain Vol, into subdomains $\mathrm{Vol}^{(m)}, m=1,2, \ldots, N$, where $N$ is the number of elements [1]. A two-dimensional quadrilateral nine node element as shown in Fig. 1 is considered. In the element, all variables are interpolated quadratically. Let the prescribed Dirichlet boundary conditions on the surface $S_{u}$ be $\boldsymbol{g}(t)$ where the vector $\boldsymbol{g}(t)$ contains the specified function of the solution on the boundary $S_{u}$. Then the solution lies in $V_{h}$ and the weighting functional space is $W_{h}$

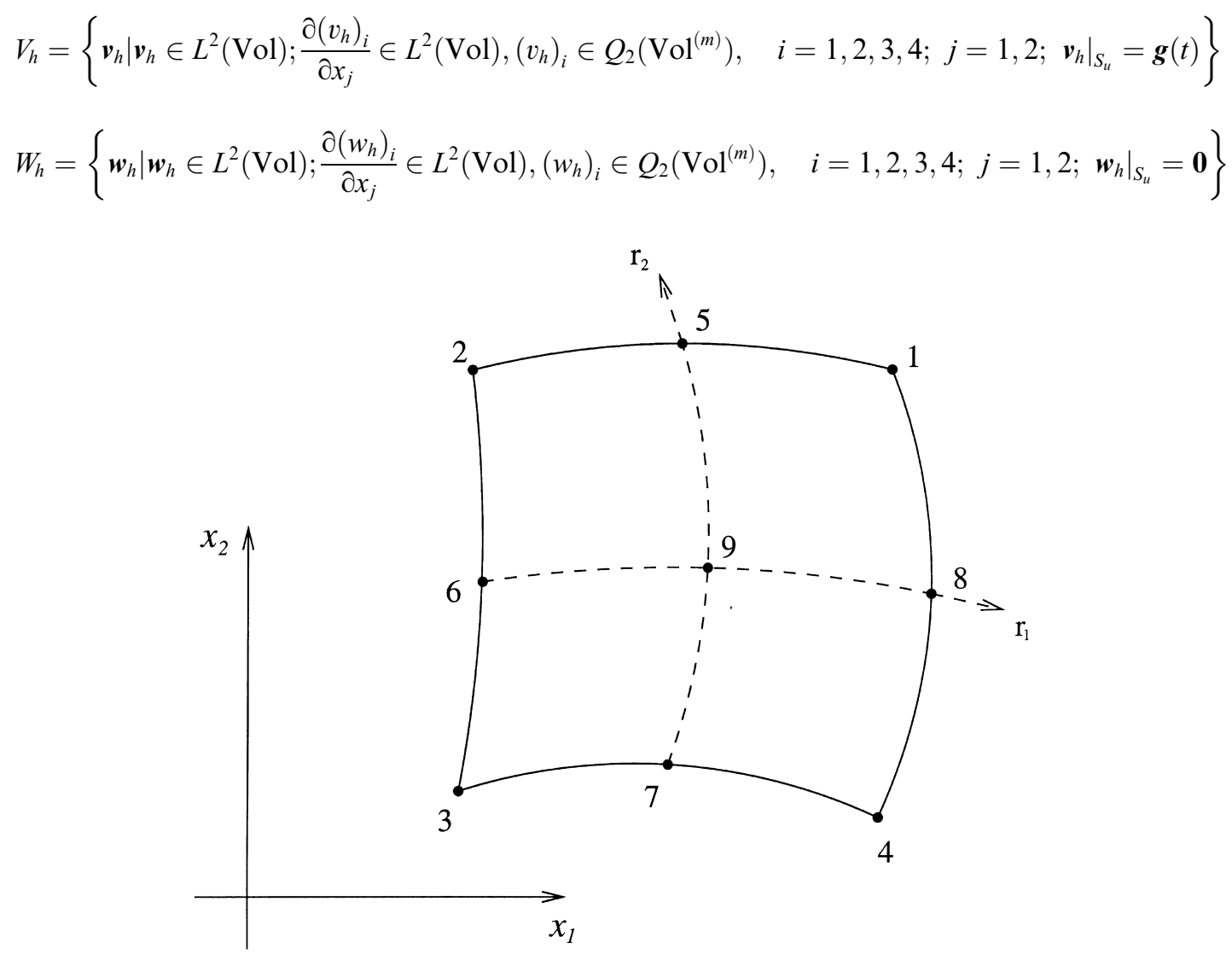

Fig. 1. Nine-node quadrilateral element used for planar flows. 
where $Q_{2}\left(\mathrm{Vol}^{(m)}\right)$ denotes the biquadratic function in the reference element $m$; and $L^{2}(\mathrm{Vol})$ is the space of square integrable functions in the volume, "Vol", of the body considered,

$$
L^{2}(\mathrm{Vol})=\left\{\boldsymbol{w} \mid \boldsymbol{w} \text { is defined in } \mathrm{Vol} \text { and } \int_{\mathrm{Vol}}\left(\sum_{i=1}^{4}\left(w_{i}\right)^{2}\right) \mathrm{dVol}=\|\boldsymbol{w}\|_{L^{2}(\mathrm{Vol})}^{2}<+\infty\right\}
$$

\subsection{Weighted residual formulation}

The finite element formulation of the Navier-Stokes equations is:

Find $\boldsymbol{U}_{h} \in V_{h}$ such that for all $\boldsymbol{W}_{h} \in W_{h}$ the following variational equation is satisfied:

$$
\begin{aligned}
\int_{\mathrm{Vol}} & {\left[\boldsymbol{W}_{h} \cdot\left(\boldsymbol{U}_{h, t}-\boldsymbol{S} \boldsymbol{U}_{h}\right)-\boldsymbol{W}_{h, j} \cdot \boldsymbol{A}_{j} \boldsymbol{U}_{h}+\boldsymbol{W}_{h, j} \cdot \boldsymbol{K}_{j i} \boldsymbol{U}_{h, i}\right] \mathrm{dVol} } \\
+ & \sum_{m} \int_{\mathrm{Vol}^{(m)}} \boldsymbol{W}_{h, k k} \cdot \boldsymbol{A}_{k} \tau_{k} \boldsymbol{A}_{k} \boldsymbol{U}_{h, k k} \mathrm{dVol}^{(m)}+\sum_{m} \int_{\mathrm{Vol}(m)} v_{k} \boldsymbol{W}_{h, k} \cdot \boldsymbol{U}_{h, k} \mathrm{dVol}^{(m)} \\
= & \int_{S} \boldsymbol{W}_{h}^{s} \cdot\left(-\boldsymbol{F}_{c}+\boldsymbol{F}_{d}\right) \mathrm{d} S
\end{aligned}
$$

Here we have used that $\boldsymbol{F}_{j}=\boldsymbol{A}_{j} \boldsymbol{U}$. The vector $\boldsymbol{W}_{h}^{s}$ is the weighting function evaluated on the surface $S$, where $S$ is the entire boundary surface, and $\boldsymbol{F}_{c}$ and $\boldsymbol{F}_{d}$ are prescribed boundary values corresponding to the convective and diffusive terms

$$
\begin{aligned}
\boldsymbol{F}_{c}=\boldsymbol{F}_{j} n_{j}= & {\left[\begin{array}{c}
\rho v_{j} n_{j} \\
\rho v_{1} v_{j} n_{j}+(\gamma-1) \rho\left(E-\frac{1}{2} v_{k}^{2}\right) \delta_{1 j} n_{j} \\
\rho v_{2} v_{j} n_{j}+(\gamma-1) \rho\left(E-\frac{1}{2} v_{k}^{2}\right) \delta_{2 j} n_{j} \\
\rho E v_{j} n_{j}+(\gamma-1) \rho v_{j}\left(E-\frac{1}{2} v_{k}^{2}\right) n_{j}
\end{array}\right], } \\
\boldsymbol{F}_{d}=\boldsymbol{G}_{j} n_{j}= & {\left[\begin{array}{c}
0 \\
\lambda v_{k, k} \delta_{1 j} n_{j}+\mu\left(v_{1, j}+v_{j, 1}\right) n_{j} \\
\lambda v_{k, k} \delta_{2 j} n_{j}+\mu\left(v_{2, j}+v_{j, 2}\right) n_{j} \\
\lambda v_{j} v_{k, k} n_{j}+\mu v_{i}\left(v_{i, j}+v_{j, i}\right) n_{j}+\frac{k}{c_{v}}\left(E-\frac{1}{2} v_{k}^{2}\right)_{, j} n_{j}
\end{array}\right], }
\end{aligned}
$$

where $n_{j}$ is the $x_{j}$-direction cosine of the unit (pointed outward) boundary normal vector.

The first integral term and the surface force terms correspond to the standard Galerkin procedure applied to the compressible flow governing equations. The second integral term is the artificial diffusion term and the third integral term is the shock capturing term, both discussed in the following sections.

\subsection{Artificial diffusion}

The purpose of the artificial diffusion is to stabilize the unstable convective terms in the standard Galerkin procedure. Although the quadratic element is "more stable" than the linear element, it still requires artificial diffusion (that is, upwinding) to reduce oscillations. Oden et al. used an artificial diffusion of the form $-\left(c \Delta t h^{2}\left|\partial v_{i} / \partial x_{i}\right| \boldsymbol{U}_{, x_{i}}\right)_{x_{i}}$ for their $h-p$ discretization schemes where $c, \Delta t, h$ are a problem-adjustable parameter, the time increment and the element size, respectively. In our work, the artificial diffusion term for the quadratic element is given by

$$
\sum_{m} \int_{\mathrm{Vol}^{(m)}} \boldsymbol{W}_{h, k k} \cdot\left(\boldsymbol{A}_{k} \tau_{k} \boldsymbol{A}_{k}\right) \boldsymbol{U}_{h, k k} \mathrm{dVol}^{(m)},
$$

The motivation for the upwind term in Eq. (10) is given in Appendix A. Note that high-order derivatives are used in order to achieve a higher order accuracy in the upwind method.

The above upwind technique applies an artificial diffusion in all directions instead of only in the streamline direction as in the SUPG technique, but the magnitude of upwinding in the $k$-direction depends 
on the convective matrix $\boldsymbol{A}_{k}$. Our numerical experiments have shown that applying the artificial diffusion in all directions gives a more stable numerical result than when the artificial diffusion is only applied in the streamline direction, and crosswind diffusion is insignificant. This is because a high order upwind method is employed.

The value of $\tau_{k}$ for the artificial diffusion is of the form used in finite difference solutions,

$$
\tau_{k}=\frac{1}{9}\left(\left|\frac{\partial x_{k}}{\partial \mathbf{r}}\right|\right)^{3}\left|\boldsymbol{A}_{k}\right|^{-1}
$$

where $\mathbf{r}$ denotes the coordinates in the natural coordinate system of the element. For the two-dimensional case

$$
\left|\frac{\partial x_{k}}{\partial \mathbf{r}}\right|=\sqrt{\left(\frac{\partial x_{k}}{\partial r_{1}}\right)^{2}+\left(\frac{\partial x_{k}}{\partial r_{2}}\right)^{2}}
$$

and we define

$$
\left|\boldsymbol{A}_{k}\right|^{-1}=\boldsymbol{X}_{k}\left|\Lambda_{k}\right|^{-1} \boldsymbol{X}_{k}^{-1}
$$

where $\boldsymbol{X}_{k}$ stores the eigenvectors of the matrix $\boldsymbol{A}_{k}$ and $\Lambda_{k}$ is a diagonal matrix with the corresponding eigenvalues. The factor $1 / 9$ is chosen by considering the $1-\mathrm{D}$ convection-diffusion problem. With the factor $1 / 9$, the artificial diffusion results into full upwinding corresponding to the corner nodes of the elements.

To obtain an understanding of how the artificial diffusion term affects the Galerkin formulation, we consider the finite element method applied to the one-dimensional steady-state Euler equation,

$$
-\int_{\mathrm{Vol}} \boldsymbol{W}_{h, 1} \cdot \boldsymbol{A}_{1} \boldsymbol{U}_{h} \mathrm{dVol}+\sum_{m} \int_{\mathrm{Vol}(m)} \boldsymbol{W}_{h, 11} \cdot \boldsymbol{A}_{1} \tau_{1} \boldsymbol{A}_{1} \boldsymbol{U}_{h, 11} \mathrm{dVol}^{(m)}=-\int_{S} \boldsymbol{W}_{h}^{s} \cdot \boldsymbol{F}_{c} \mathrm{~d} S .
$$

For a uniform mesh, $\Delta x$ is constant so that for each of the elements

$$
\tau_{1}=\frac{1}{9}\left(\frac{\Delta x}{2}\right)^{3}\left|A_{1}\right|^{-1}
$$

Combining Eqs. (14) and (15), we obtain

$$
\sum_{m} \int_{\operatorname{Vol}^{(m)}}\left\{-\boldsymbol{W}_{h, 1} \cdot \boldsymbol{A}_{1} \boldsymbol{U}_{h}+\boldsymbol{W}_{h, 11} \cdot \frac{1}{9}\left(\frac{\Delta x}{2}\right)^{3}\left|\boldsymbol{A}_{1}\right| \boldsymbol{U}_{h, 11}\right\} \mathrm{dVol}^{(m)}=-\int_{S} \boldsymbol{W}_{h}^{s} \cdot \boldsymbol{F}_{c} \mathrm{~d} S,
$$

where for a smooth function $\boldsymbol{U}$, the second term (the artificial diffusion term) approaches zero with third order as $\Delta x \rightarrow 0$. Hence for a smooth function $\boldsymbol{U}$, the parabolic element with the third order artificial diffusion term can be expected to give more accurate results than the SUPG method using the linear element.

\subsection{Shock capturing}

The shock capturing term is designed to reduce oscillations in the vicinity of shocks and other discontinuities. In the formulation considered, the shock capturing term is

$$
\sum_{m} \int_{\mathrm{Vol}^{(m)}} v_{k} \boldsymbol{W}_{h, k} \cdot \boldsymbol{U}_{h, k} \mathrm{dVol}^{(m)}
$$

where $v_{k}$ is a tuned variable. This shock capturing term applies an artificial diffusion in all directions with the magnitude in the $x_{k}$-direction proportional to $v_{k}$. We wish to have a value of $v_{k}$ that is small at a reasonable distance from the shock and sufficiently large in the vicinity of the shock, and use

$$
v_{k}=\frac{1}{4} \frac{h_{k}\left\|h_{j}^{2} \boldsymbol{A}_{j} \boldsymbol{U}_{h, j j}\right\|}{\left\|\boldsymbol{U}_{h}\right\|},
$$




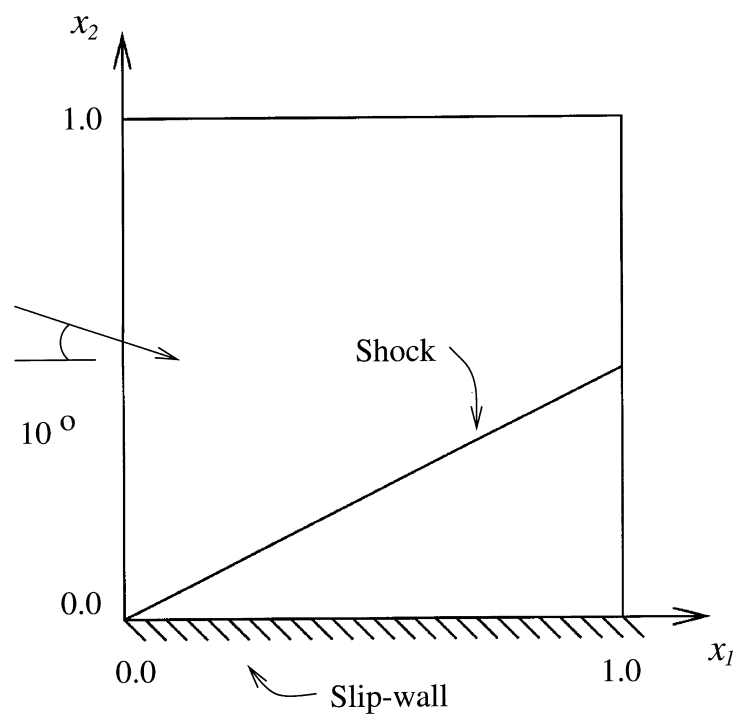

Fig. 2. Two dimensional shock problem.

where $h_{l}$ is the $x_{l}$-direction "length" of the element,

$$
h_{l}=\frac{1}{\sqrt{\left(\frac{1}{2} \frac{\partial r_{1}}{\partial x_{l}}\right)^{2}+\left(\frac{1}{2} \frac{\partial r_{2}}{\partial x_{l}}\right)^{2}}} .
$$

The value $1 / 4$ is obtained by choosing the factor that gives the best shock solution of the test problem considered at the end of this section. If, instead, the factors $1 / 3$ or $1 / 6$ are used, only a slightly different shock solution is observed. This shock capturing term has the form given by Beau et al. [8] (derived from the shock capturing term proposed by Hughes et al. [16,22]) for linear elements, but the difference lies in the definition of $v_{k}$.

Away from the shock where the finite element solution is smooth, $\boldsymbol{U}_{h, j j}$ is not large (the magnitude depends of course on the problem considered) and $v_{k}$ is small for a small element size. In those regions, the shock capturing term gives third-order accuracy since $\boldsymbol{U}_{h, j j}$ weakly depends on the mesh size. On the other hand, in the vicinity of a shock, the finite element solution gives a large value $\boldsymbol{U}_{h, j j}$, hence $v_{k}$ is large and the shock capturing term smoothens the solution. Near a shock, the shock capturing term gives first-order accuracy because on the shock $\boldsymbol{U}_{h, j j}$ is a function of the mesh size which cancels the $h_{j}^{2}$ term in the $v_{k}$ definition.

To show the capability of the shock capturing method, consider the test problem in Fig. 2 [6,26]. The two-dimensional steady-state problem contains an oblique shock. At the inlet on the left and upper boundaries, the convective fluxes are prescribed corresponding to the condition

$$
M=2, \rho=1, v_{1}=\cos 10^{\circ}, v_{2}=-\sin 10^{\circ} .
$$

The convective fluxes are imposed at the left and upper boundaries instead of imposing the Dirichlet boundary condition in order to avoid the inconsistency in the boundary conditions at the lower-left corner where the slip-wall condition is imposed. On the right boundary, no variable is prescribed. The uniform and distorted meshes shown in Fig. 3 were used. The finite element solutions are shown in Fig. 4. The finite element method using the quadratic element gives reasonable results for both meshes.

The plots of density distributions along $x_{1}=1$ using both meshes are shown in Fig. $5 .{ }^{1}$ This figure shows that the shock is captured within at most four elements with two elements containing the high gradient of

\footnotetext{
${ }^{1}$ Solutions are presented by simply connecting nodal values.
} 


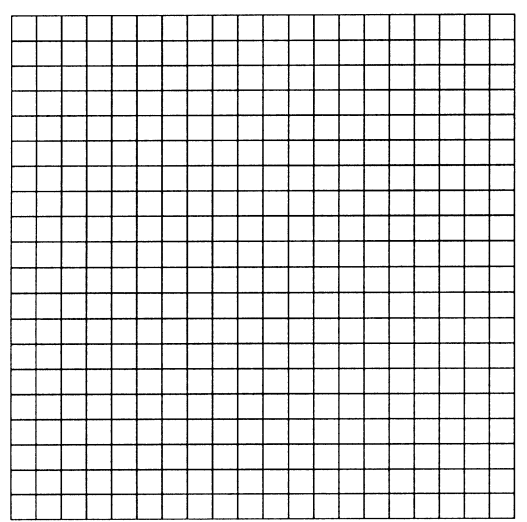

(a)

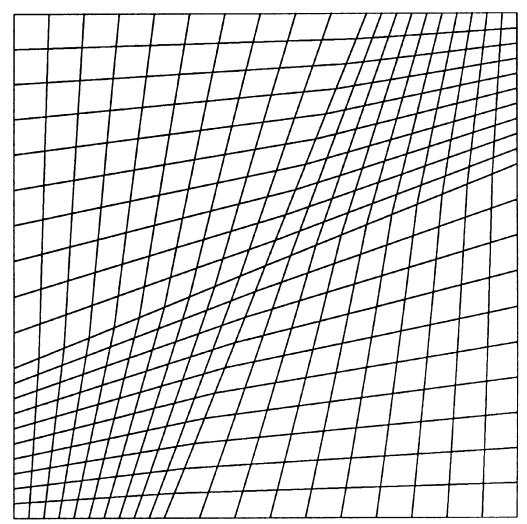

(b)

Fig. 3. Meshes used for the two-dimensional shock problem (a) uniform mesh, (b) distorted mesh.

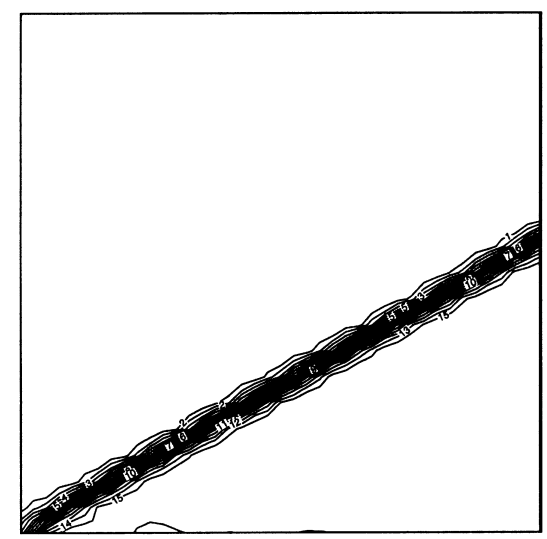

(a)

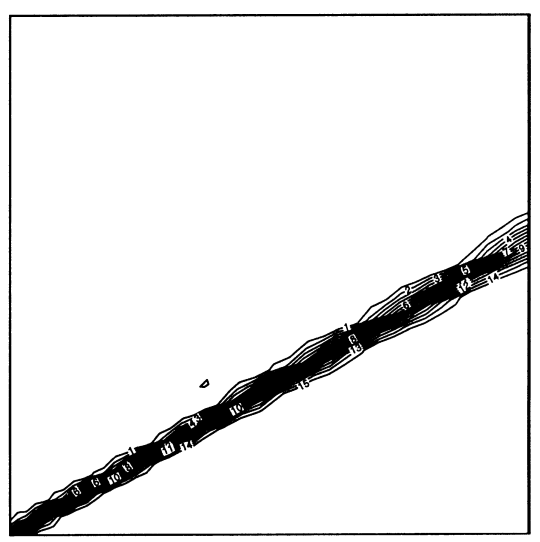

(b)

Fig. 4. Contour plot of density of the finite element solution using (a) uniform mesh, (b) distorted mesh.

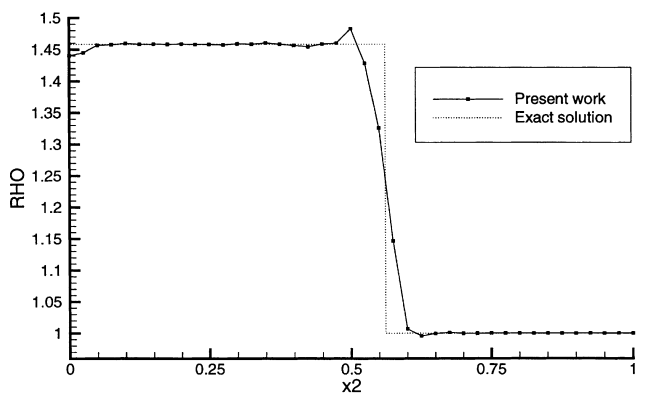

(a)

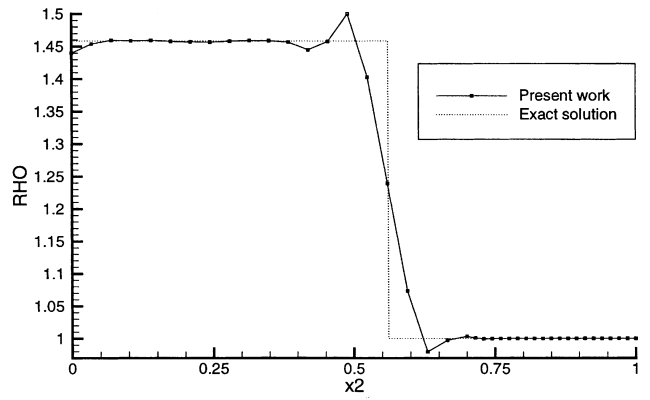

(b)

Fig. 5. Density distribution along $x_{1}=1.0$ using (a) uniform mesh, (b) distorted mesh.

the jump variable and the other two elements giving a smooth transition of the solution. The solutions also show some overshoot at the shock front which is of course not desirable.

Fig. 4 shows that the mesh with distorted elements gives a better solution. This is the case, because the element distribution is favorable to the shock in the lower-left corner where the shock starts. Namely, the 
mesh is finer in this corner, and of course the smaller the elements used to capture the shock, the better is the solution.

\section{Numerical examples}

In this section, we present numerical examples to demonstrate the stability and accuracy of the finite element method using the quadratic element.

\subsection{Supersonic flow over a bump}

The problem of supersonic flow over a bump is described in Fig. 6. In this problem, a bump is placed in a wind tunnel and the flow is assumed to be frictionless. The bump arc is described by

$$
x_{2}=0.04\left(1-4\left(x_{1}-1.5\right)^{2}\right) \quad 1 \leqslant x_{1} \leqslant 2 .
$$

The free-stream flow has the following condition

$$
M=1.4, \rho=1, v_{1}=1, v_{2}=0 .
$$

The fluid properties are assumed to be constant with $\gamma=1.4, c_{v}=715, k=0, \mu=0$. The domain is discretized into a mesh of $15 \times 46$ elements where the velocity boundary condition on the curved slip wall is specified as explained by Wang and Bathe [24]. The calculated nodal pressure distribution using the quadratic element is shown in Fig. 7. For comparison, the ADINA-F solution for this problem is shown in Fig. 8 [25].

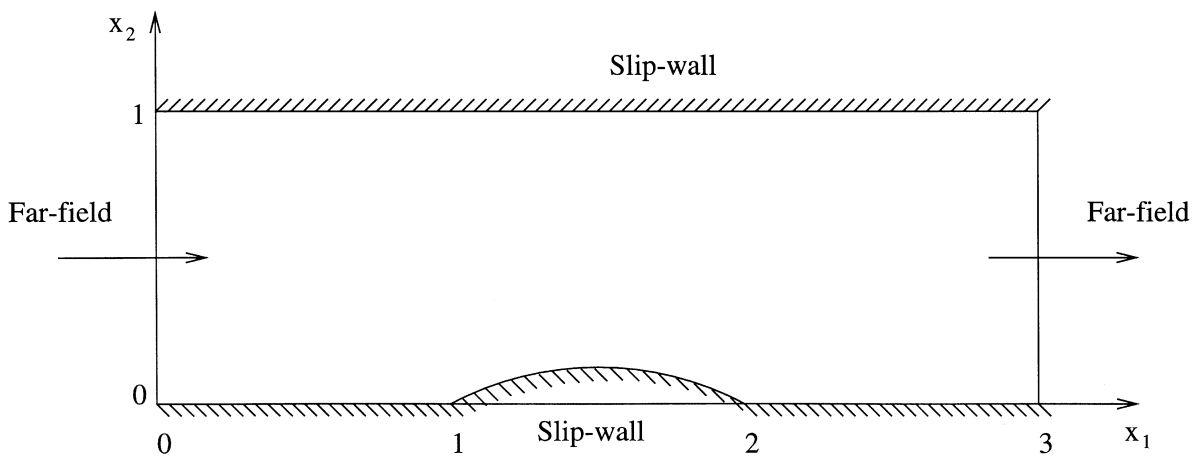

Fig. 6. Supersonic flow over a bump problem.
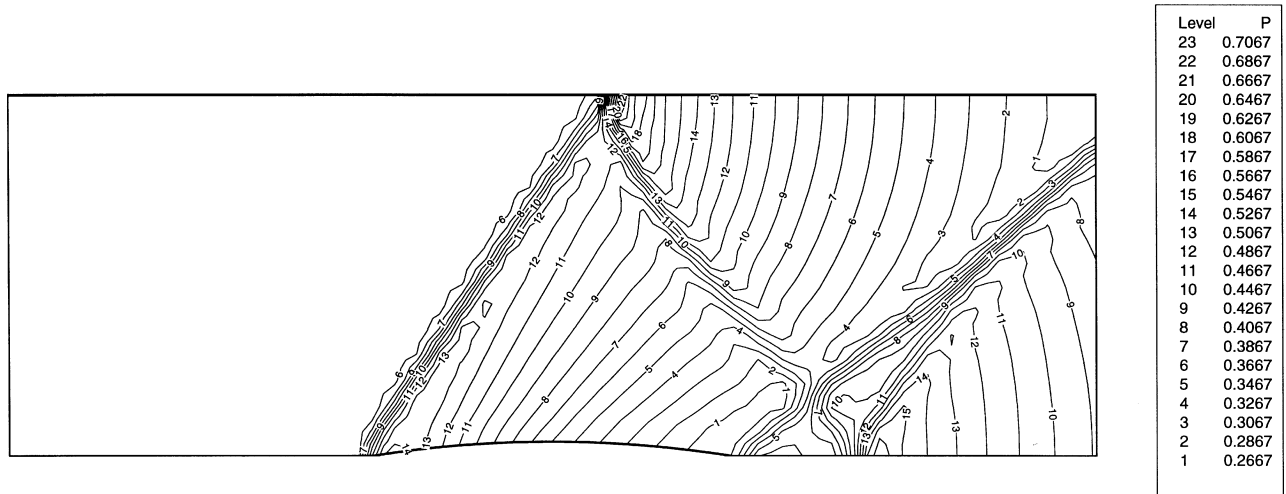

Fig. 7. Nodal pressure solution of the supersonic flow over a bump problem. 


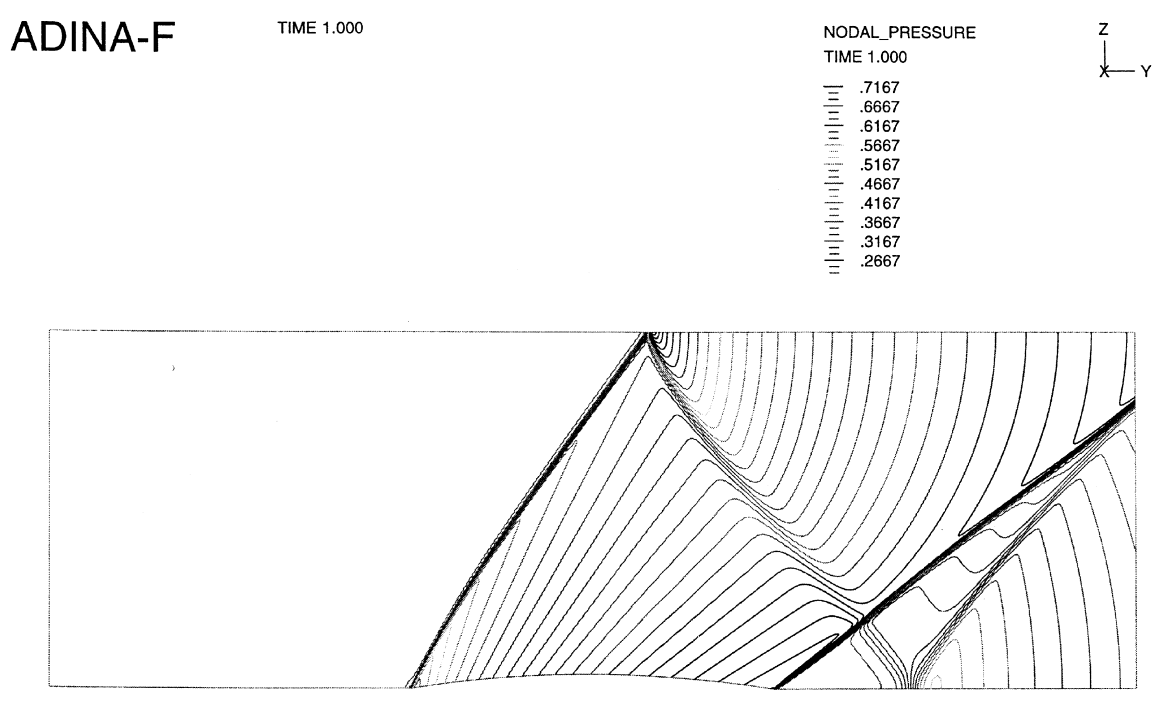

Fig. 8. Nodal pressure solution of the supersonic flow over a bump problem using ADINA-F.

The solution shows that a shock originates from the leading edge of the bump. As the shock reaches the upper boundary where the slip-wall condition is applied, the shock is reflected and then as the reflected shock hits the lower boundary, to the right of the trailing edge of the bump, it is reflected again. The reflected shock is interacting with the shock developed at the trailing edge of the bump.

The density distributions along $x_{2}=1$ and along the center of the channel are shown in Fig. 9. The solution by Beau et al. [8] using a fine mesh, $60 \times 184$, and the solution using ADINA-F with a very fine mesh are also shown for comparison. From Fig. 9, we see that the quadratic element solution is close to the prediction presented by Beau et al. and the ADINA-F solution. The quadratic element solution for the shock originating from the leading edge is close to the comparison solutions in terms of the shock magnitude and location, and the same holds for the calculated reflected shock. However, considering the solutions for the trailing edge shock, a difference in location is observed, with the quadratic element solution being closer to the ADINA-F solution than is Beau's solution. Note that in Fig. 9(b), the ADINA-F solution gives distinct shock locations for the doubly reflected leading edge shock and the trailing edge shock (around $x_{1}=2.7$ ) due to a sufficiently small element size being used.

\subsection{Natural convection problem}

To show the ability of the quadratic element to solve a very low Mach number problem, we consider the natural convection problem described in Fig. 10. To obtain the steady state solution, we used a transient analysis and iterated the solution from the initial condition to the time when the changes in the variables are small. The initial condition is given as: pressure $p=10^{5}$, temperature $\theta=300$ and velocity $v_{1}=v_{2}=0$. The fluid properties are assumed to be constant, $R=286, c_{v}=715, k=1.0, \mu=0.001$. The Rayleigh number of this problem is $R a \approx 6.5 \times 10^{5}$. We discretized the domain into a mesh of $20 \times 20$ elements with smaller elements close to the boundary to capture the boundary layers, see Fig. 11. The results of the problem using the quadratic element are shown in Figs. 12 and 13. The maximum Mach number in the solution is about 0.0005 which is reached by the fluid located at $x_{2}=0.5$ and right outside the boundary layer of the left and right side walls.

For comparison, we solve the natural convection problem using ADINA-F with the incompressible formulation and the Boussinesq approximation [27] for the density change. The calculated $x_{2}$-direction velocity and temperature distributions along $x_{2}=0.5$ are plotted in Fig. 14 for the quadratic element solution and the ADINA-F solution. The results are close to each other, especially the calculated temperature distributions. 


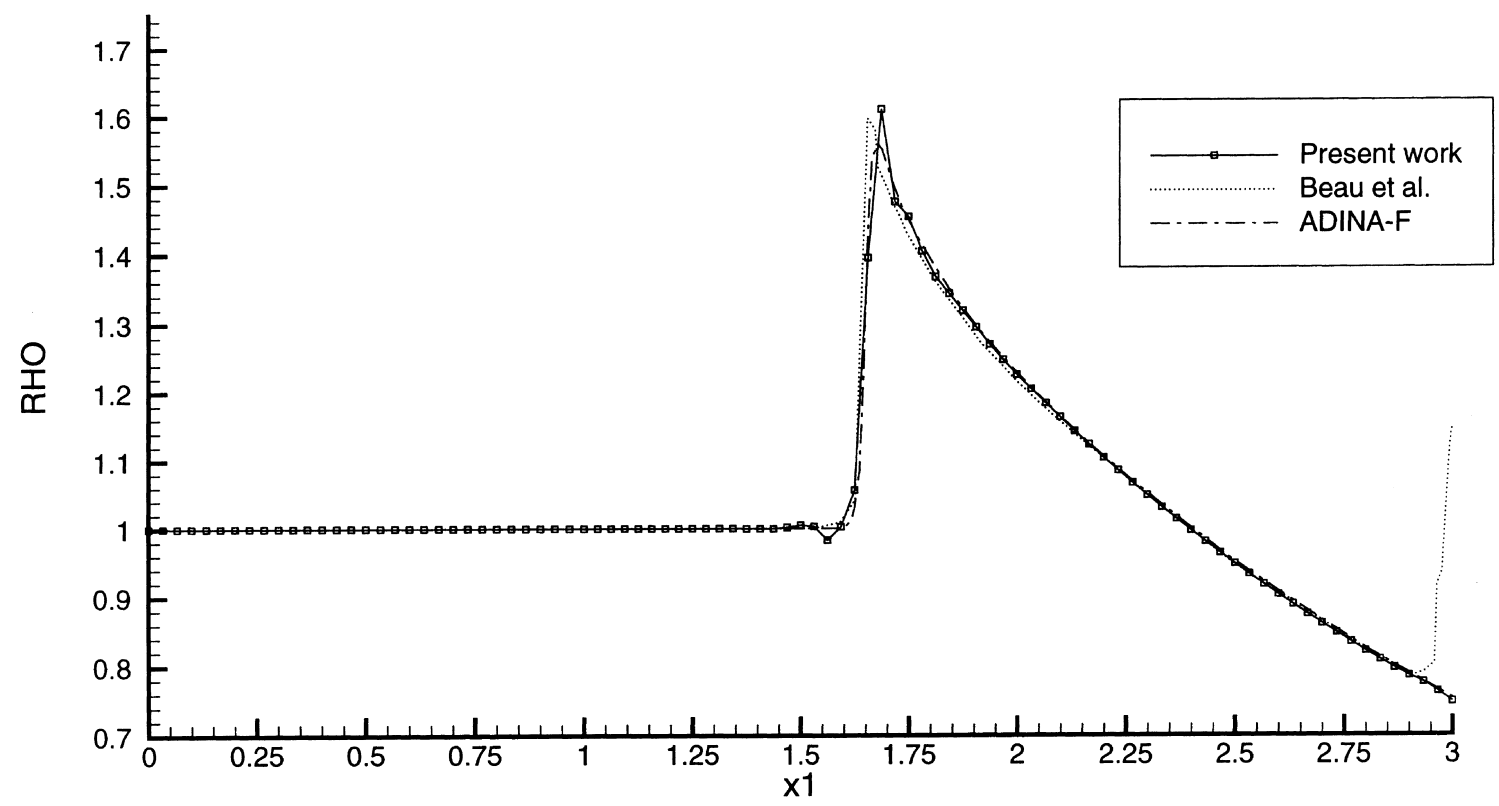

(a)

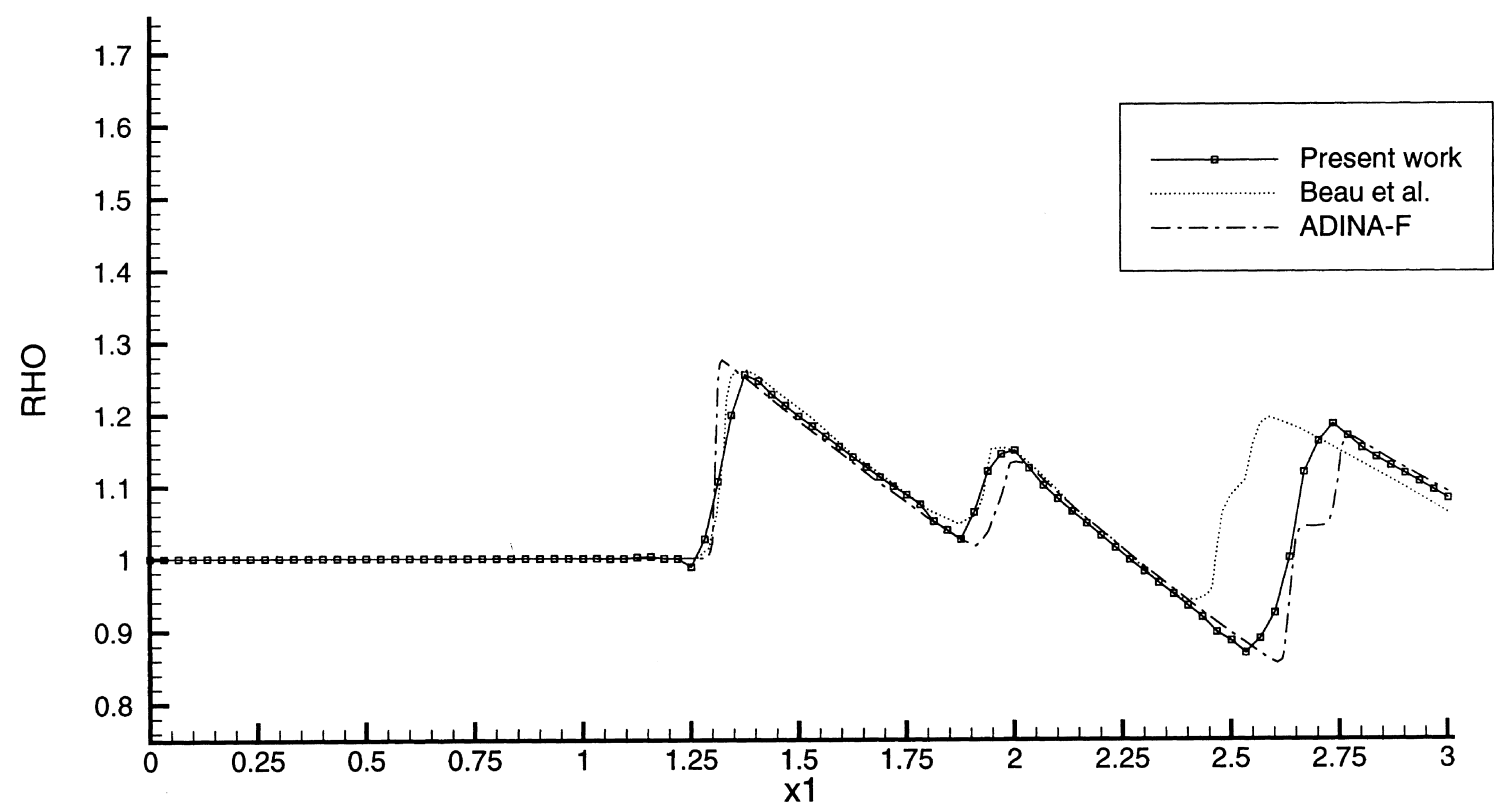

(b)

Fig. 9. Density distribution (a) along $x_{2}=1.0$, (b) along the center of the channel.

The predicted heat flux distributions on the left and right side boundaries are plotted in Fig. 15. The solution using the quadratic element is reasonably close to the solution obtained with ADINA-F.

\subsection{Supersonic flow over a flat plate}

The flow over a flat plate is solved to study the stability and accuracy of the method. The domain and boundary conditions of the 2D Navier-Stokes flow problem are shown in Fig. 16. In this problem, a Mach 


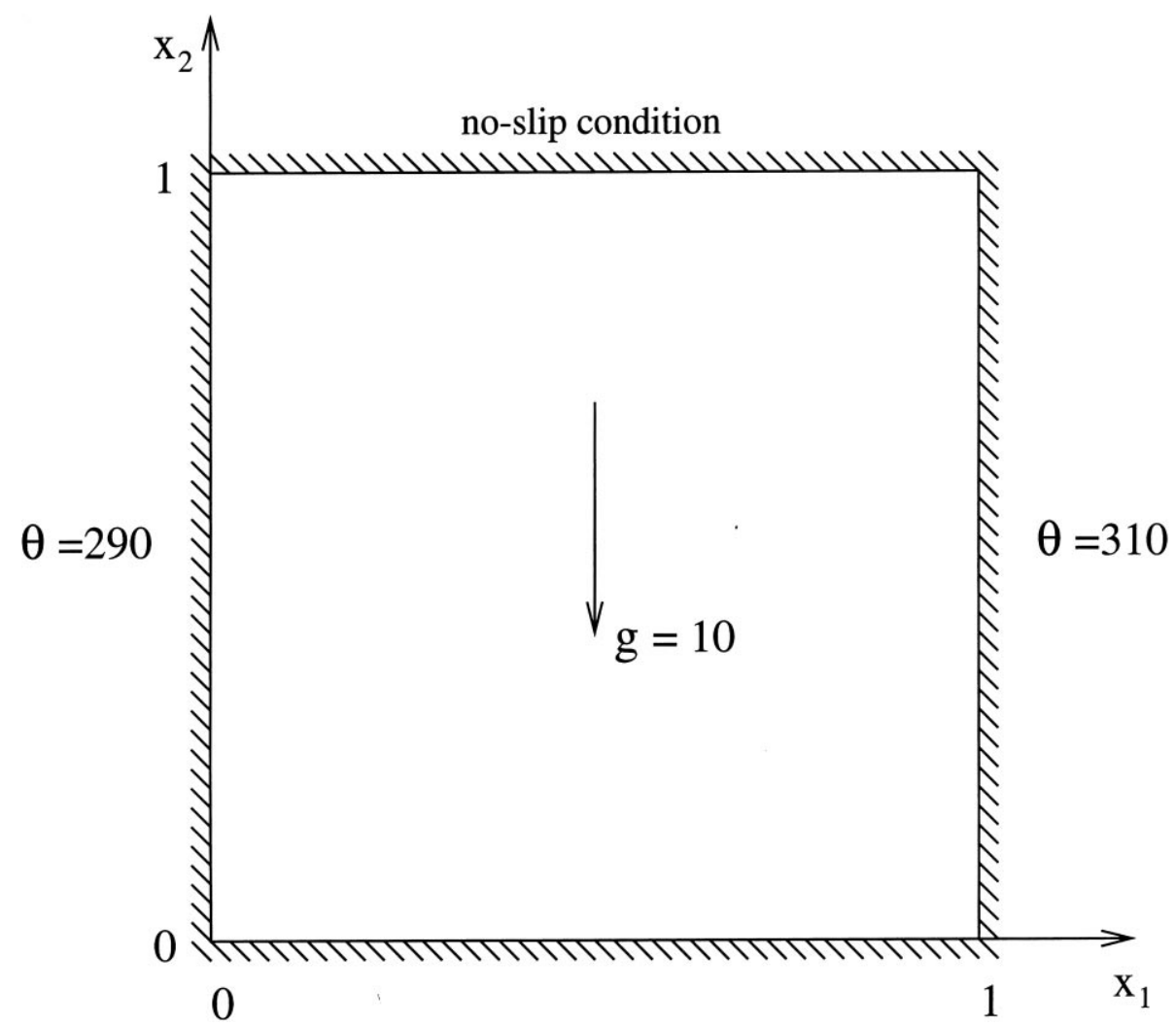

Fig. 10. The natural convection problem.

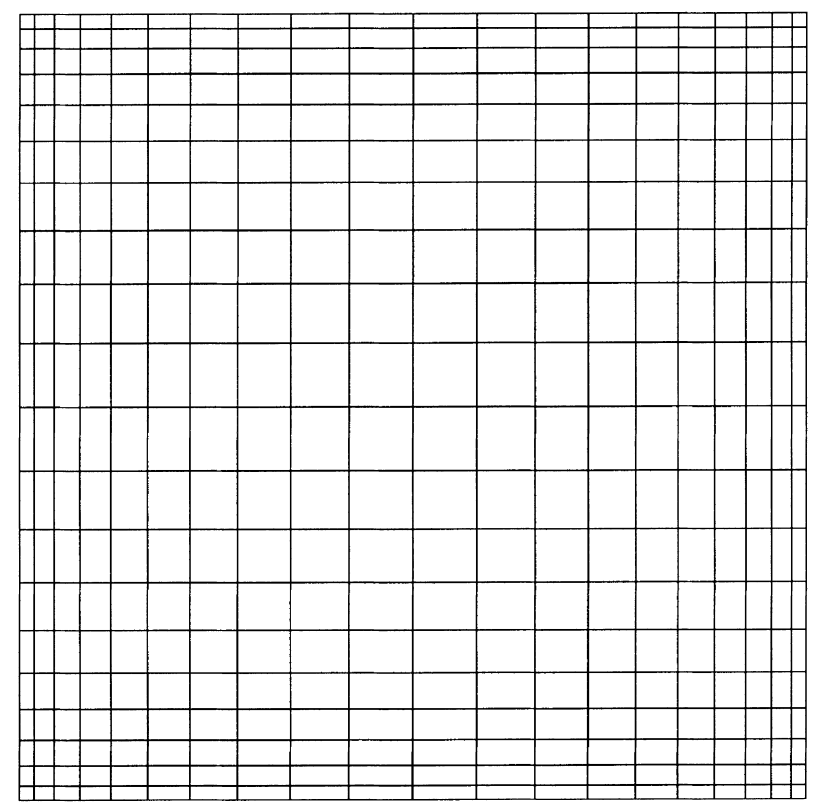

Fig. 11. The mesh used for the natural convection problem. 


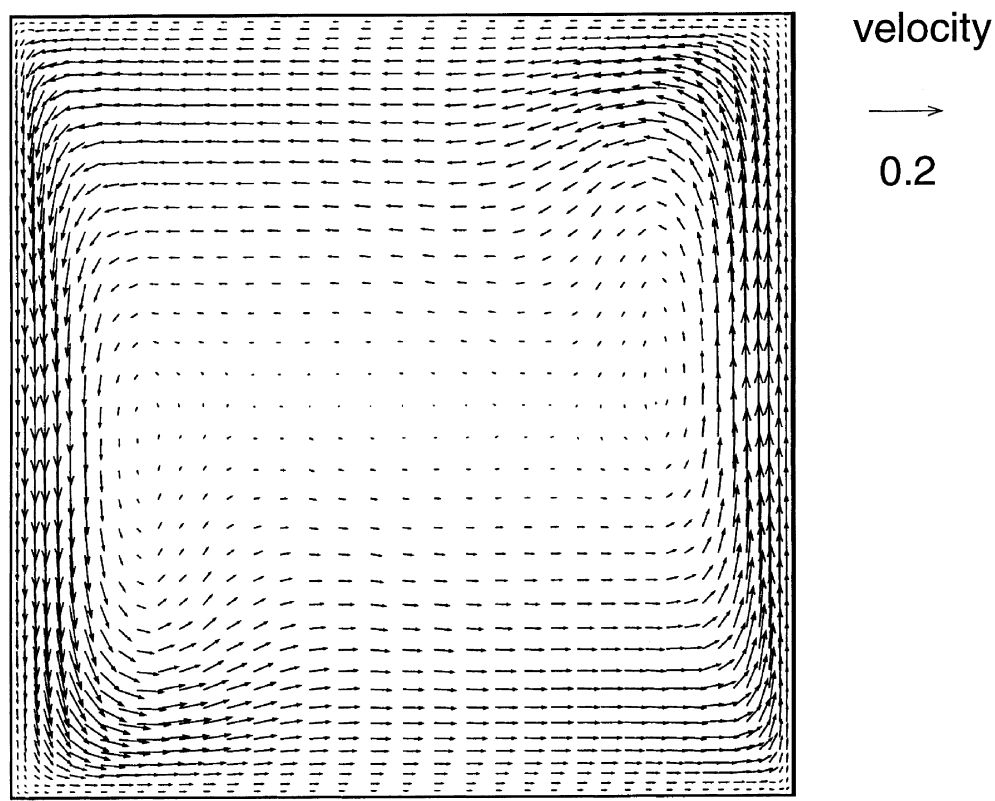

Fig. 12. The velocity vector solution of the natural convection problem.

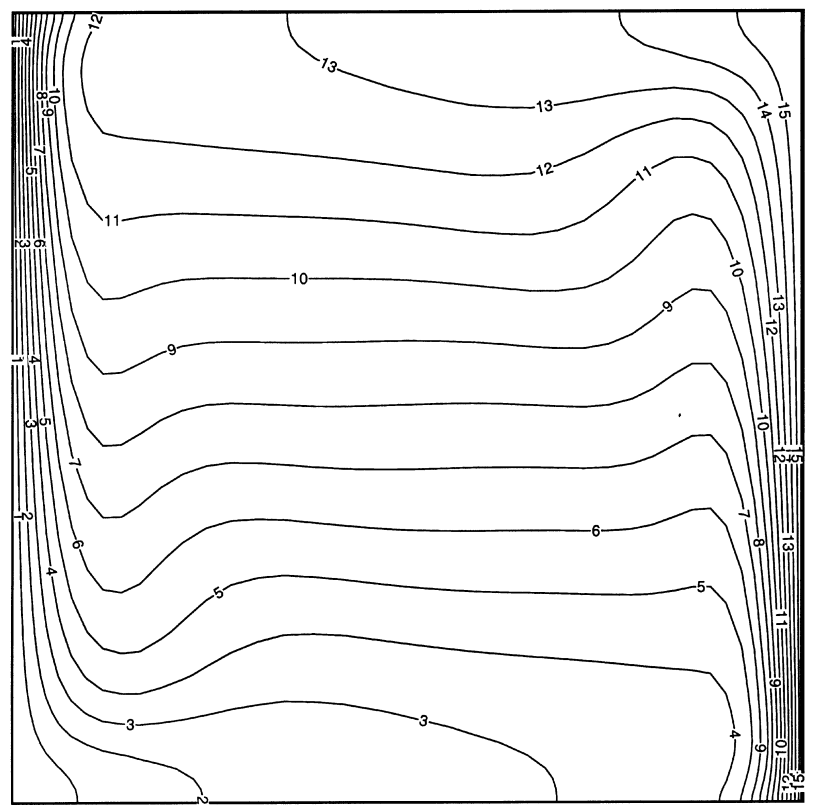

\section{temperature}

Fig. 13. The temperature distribution solution of the natural convection problem.

three flow is passing over an infinitely thin plate at zero angle of attack and a curved shock and a boundary layer are developed. The Reynolds number is $10^{3}$ based on the free stream values and the length of the plate, $L$. The fluid properties are $\gamma=1.4, R=286.62, \mu=0.0906 \theta^{1.5} /(\theta+0.0001406)$ and $k=\left(\gamma c_{\nu} \mu\right) / \operatorname{Pr}$, where $\operatorname{Pr}$ is the Prandtl number, $\operatorname{Pr}=0.72$.

The computational domain is given by $-0.2 \leqslant x_{1} \leqslant 1.2,0 \leqslant x_{2} \leqslant 0.8$, and the leading edge of the plate is located at $x_{1}=0$. The domain is discretized into a mesh of $24 \times 42$ elements with smaller elements close to 


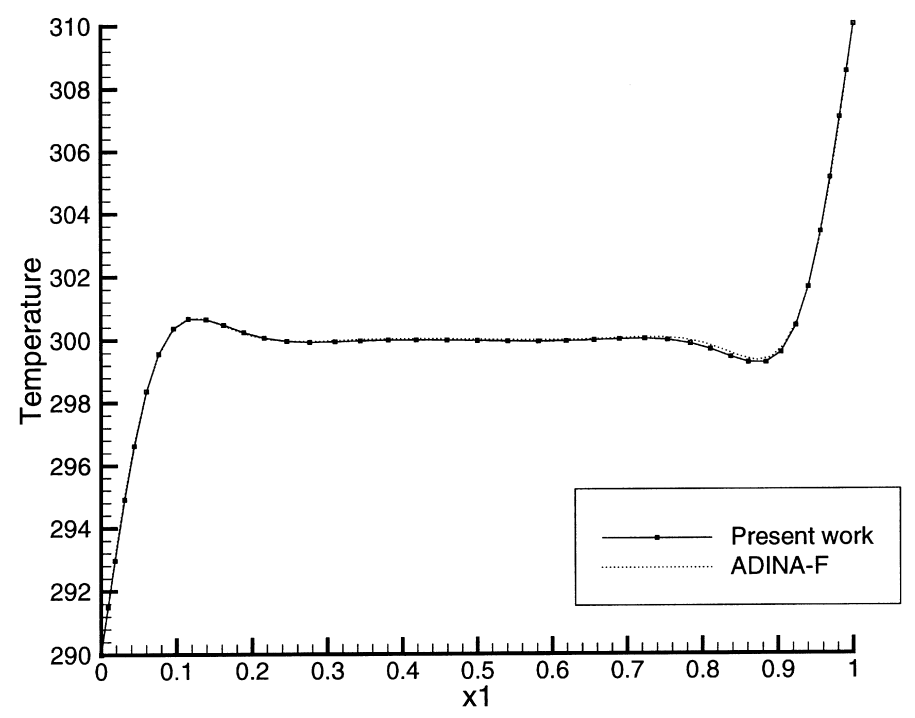

(a)

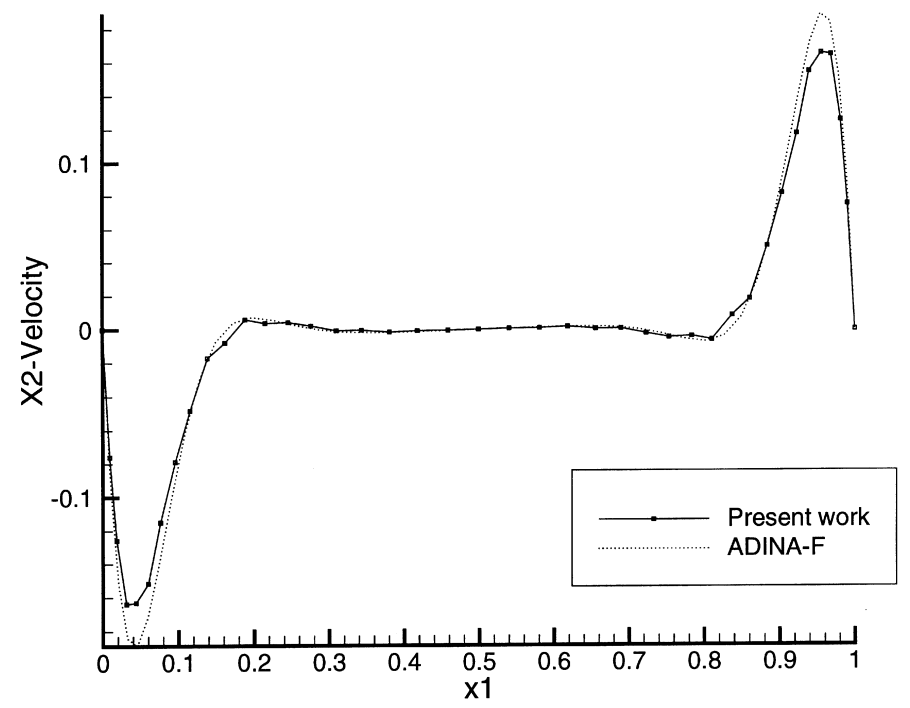

(b)

Fig. 14. The solution along $x_{2}=0.5$ (a) temperature, (b) velocity component in $x_{2}$-direction.

the leading edge of the plate. At the inflow boundary $\left(x_{1}=-0.2\right)$ and top boundary $\left(x_{2}=0.8\right)$, all four variables are prescribed with the condition $\rho=1, v_{1}=1, v_{2}=0, \theta=2.769 E-4$. Along the line $x_{2}=0$ and $x_{1}<0$, the symmetric conditions $v_{2}=\tau_{12}=q_{2}=0$ are imposed. On the plate $\left(x_{2}=0\right.$ and $\left.x_{1} \geqslant 0\right)$, the noslip condition, $v_{1}=v_{2}=0$, and the stagnation temperature, $\theta_{s}=7.754 E-4$, are prescribed. At the outflow boundary $\left(x_{1}=1.2\right)$, no variable is prescribed except the shear stress term to accommodate the boundary layer velocity profile at the right boundary,

$$
\tau_{12}=\mu\left(\frac{\partial v_{1}}{\partial x_{2}}+\frac{\partial v_{2}}{\partial x_{1}}\right)
$$

The calculated solution for the problem is shown in Fig. 17. A shock originates from the leading edge of the plate along with the development of a boundary layer. Across the shock, the density increases and the 


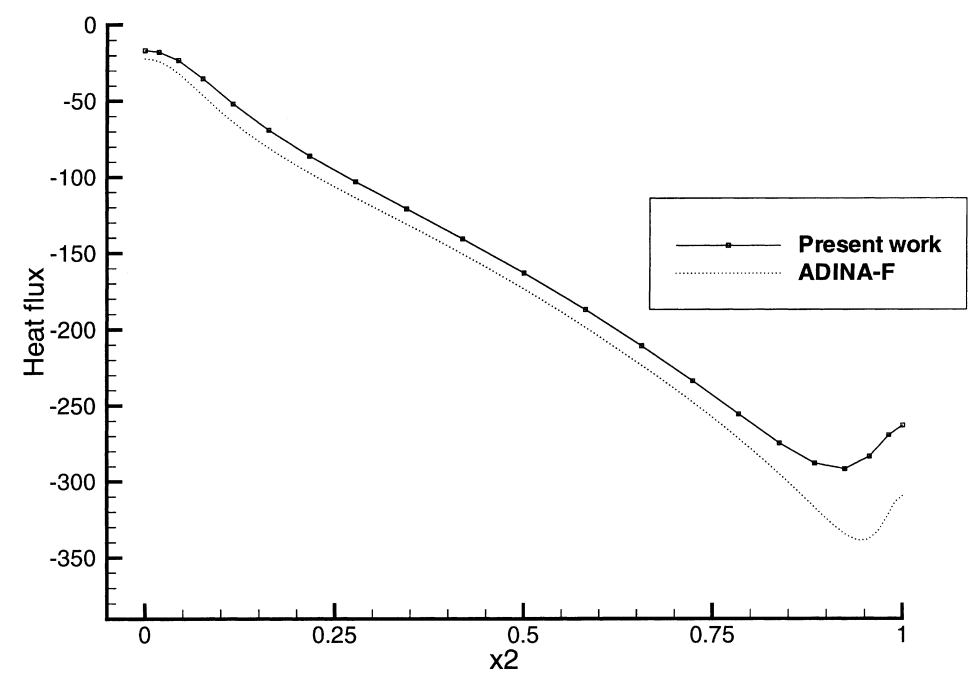

(a)

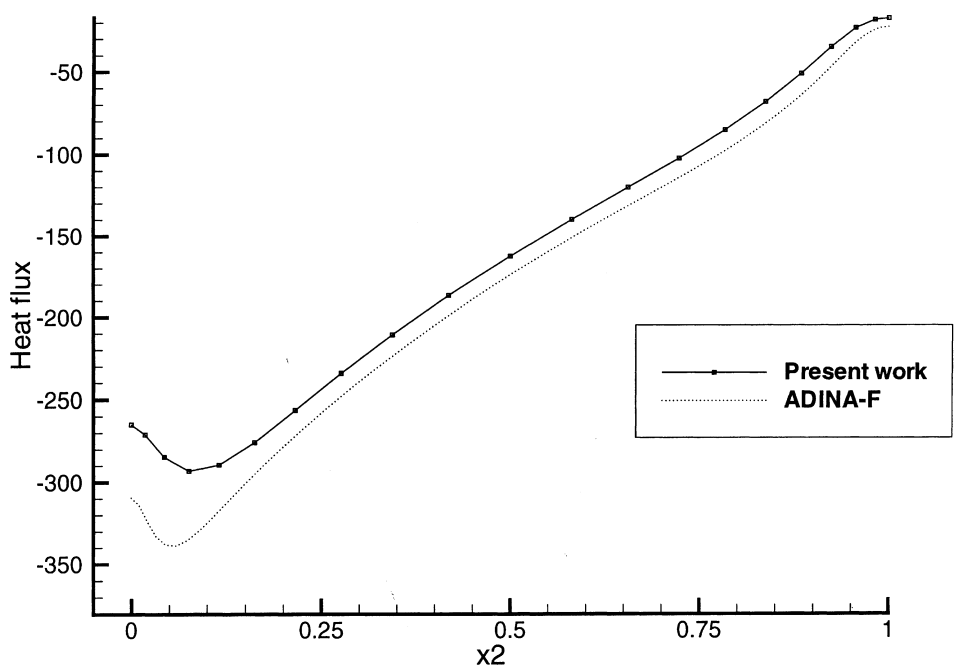

(b)

Fig. 15. The heat flux on the wall (a) left side, (b) right side.

Mach number decreases (the density has a maximum value at the leading edge point where there is a singularity). To give a comparison, Fig. 18 shows the plot of the coefficient of skin friction along the plate (defined as $C_{f}=\tau_{\text {wall }} /\left((1 / 2) \rho_{\infty} V_{\infty}^{2}\right)$, where $\rho_{\infty}$ and $V_{\infty}$ are the far-field fluid density and velocity, and $\tau_{\text {wall }}$ is the wall shear stress) and compares the computed result with the result published by Shakib et al. [26] using a very fine mesh (28672 linear elements). Although a rather coarse mesh was used, the skin friction coefficient obtained using the quadratic element is very close to the result given in Ref. [26].

\subsection{Mach 6.06 compression corner}

A Mach 6.06, Reynolds number 150000 , flow over a compression corner at an angle of $10.25^{\circ}$ is considered, see Fig. 19. The Reynolds number is calculated based on the free stream conditions and the distance from the leading edge of the plate to the corner. The fluid properties are $\gamma=1.4, R=286.62$, $\mu=0.002637 \theta^{1.5} /(\theta+0.00015324)$, and $k=\gamma c_{v} \mu / \operatorname{Pr}$, where $\operatorname{Pr}$ is the Prandtl number, $\operatorname{Pr}=0.72$. 


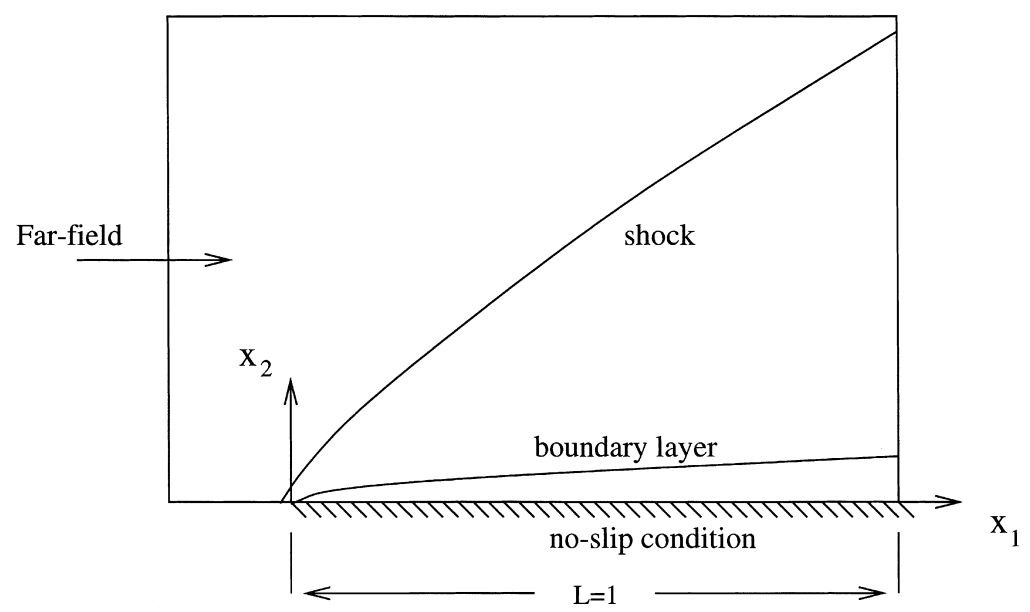

Fig. 16. Supersonic flow over a flat plate problem.
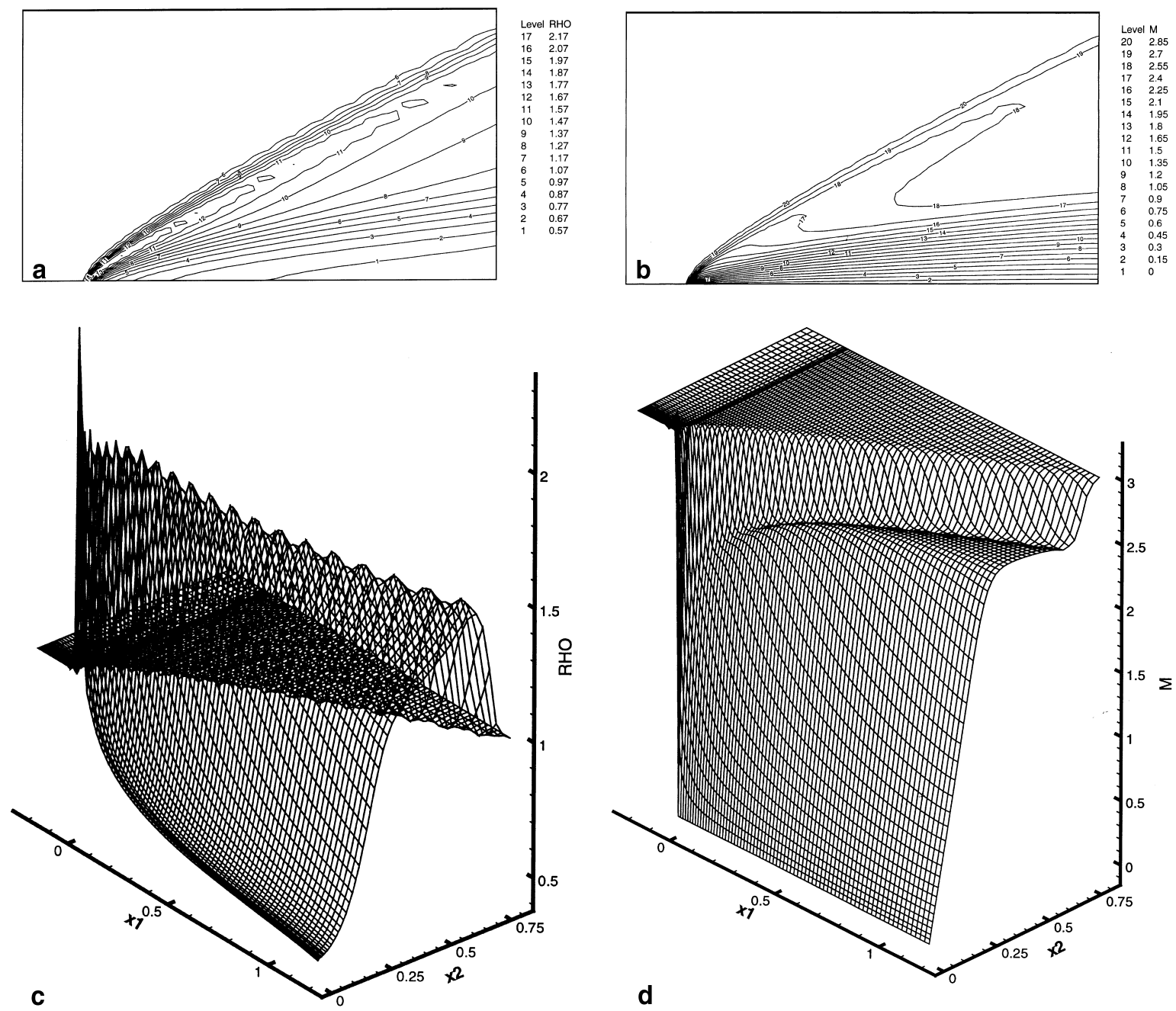

Fig. 17. Solution of the flow over a flat plate problem (a) density, (b) Mach number, (c) density distribution in 3D representation, (d) Mach number distribution in 3D representation. 


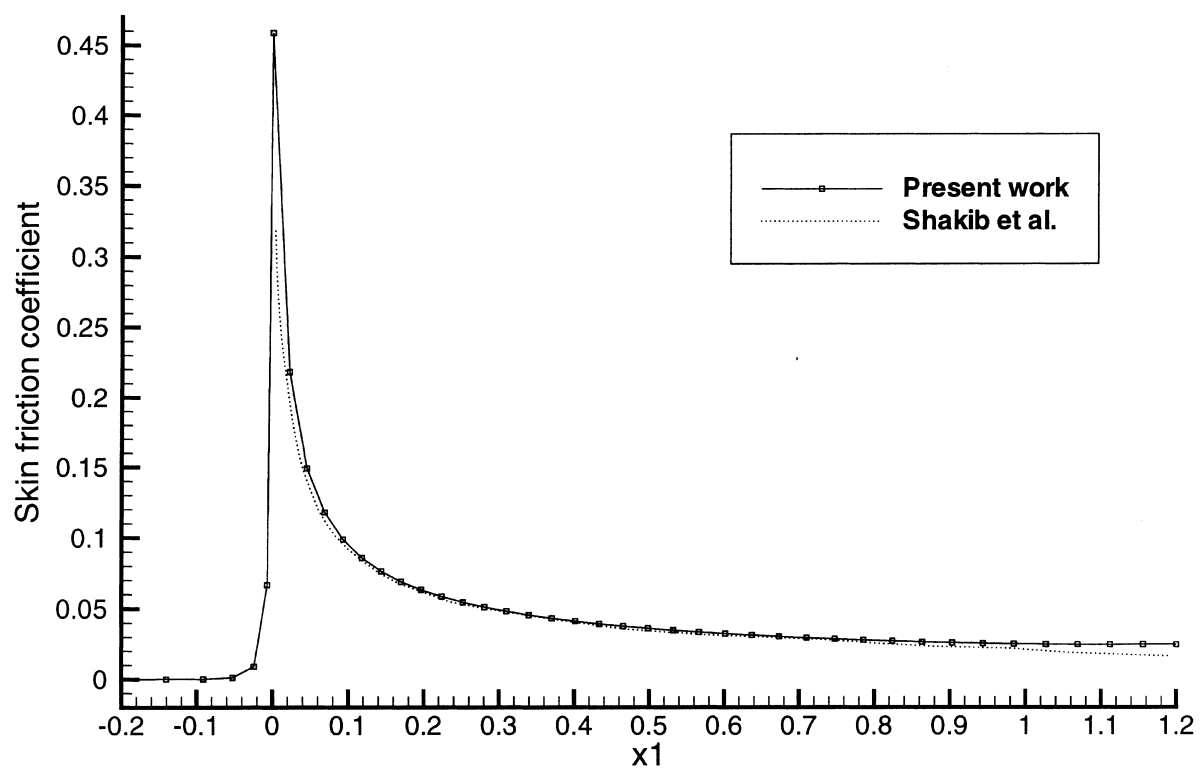

Fig. 18. Skin friction coefficient distribution along the plate.

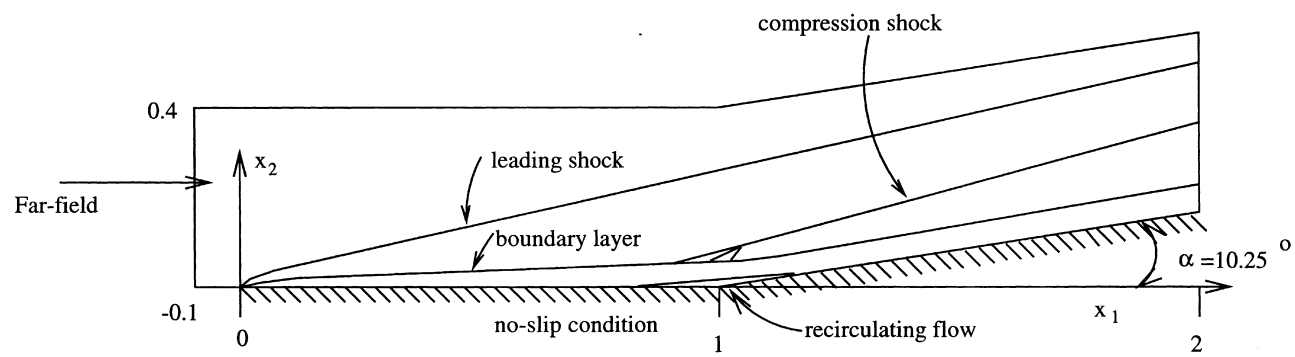

Fig. 19. Flow over a compression corner.

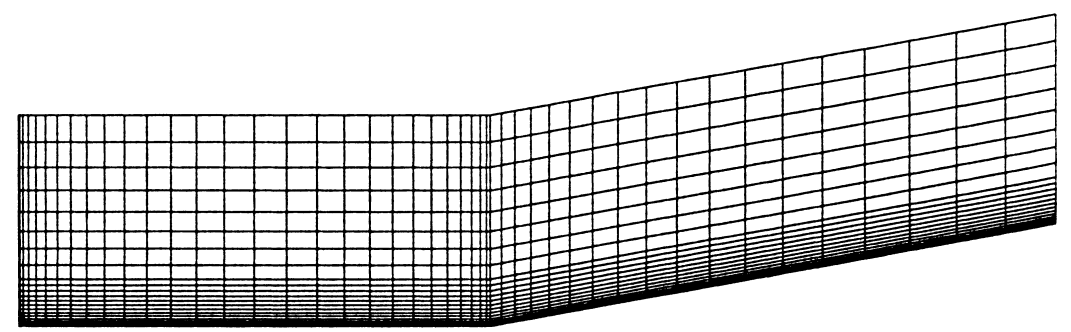

Fig. 20. The mesh used for the flow over a compression corner problem.

On the inflow and upper boundaries, all four variables are prescribed with the condition $\rho=1, v_{1}=1, v_{2}=0, \theta=6.7861 E-5$. On the plate, the no-slip condition, $v_{1}=v_{2}=0$, and the adiabatic condition are prescribed. A rather long computational domain is employed to avoid boundary effects due to the inflow and outflow conditions. The computational domain is discretized into $21 \times 46$ quadratic elements. The mesh used is shown in Fig. 20. We use many elements close to the wall to capture the boundary layer. 

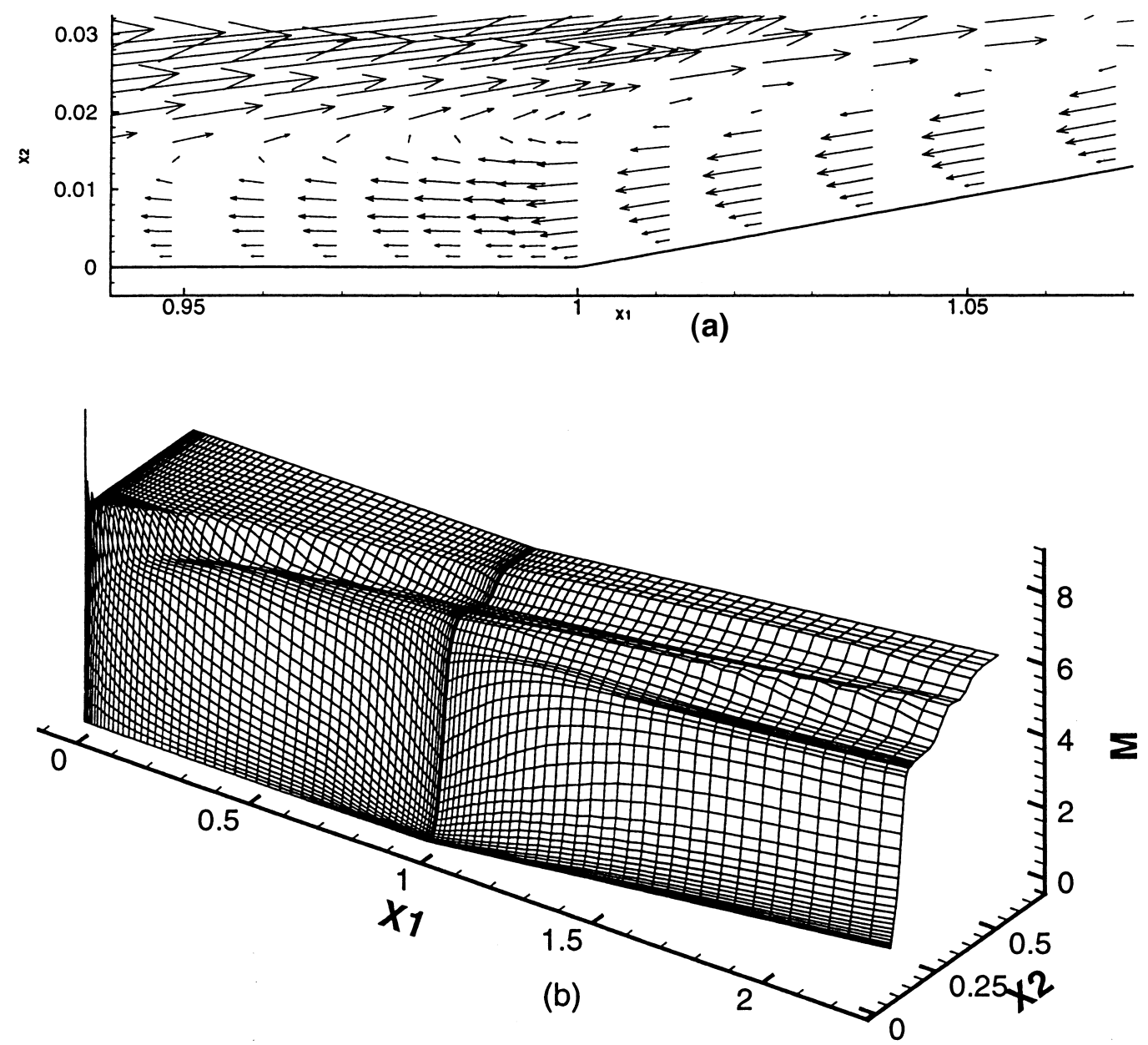

Fig. 21. Solution of the flow over a compression corner (a) backflow in the corner, (b) Mach number distribution in 3D representation.

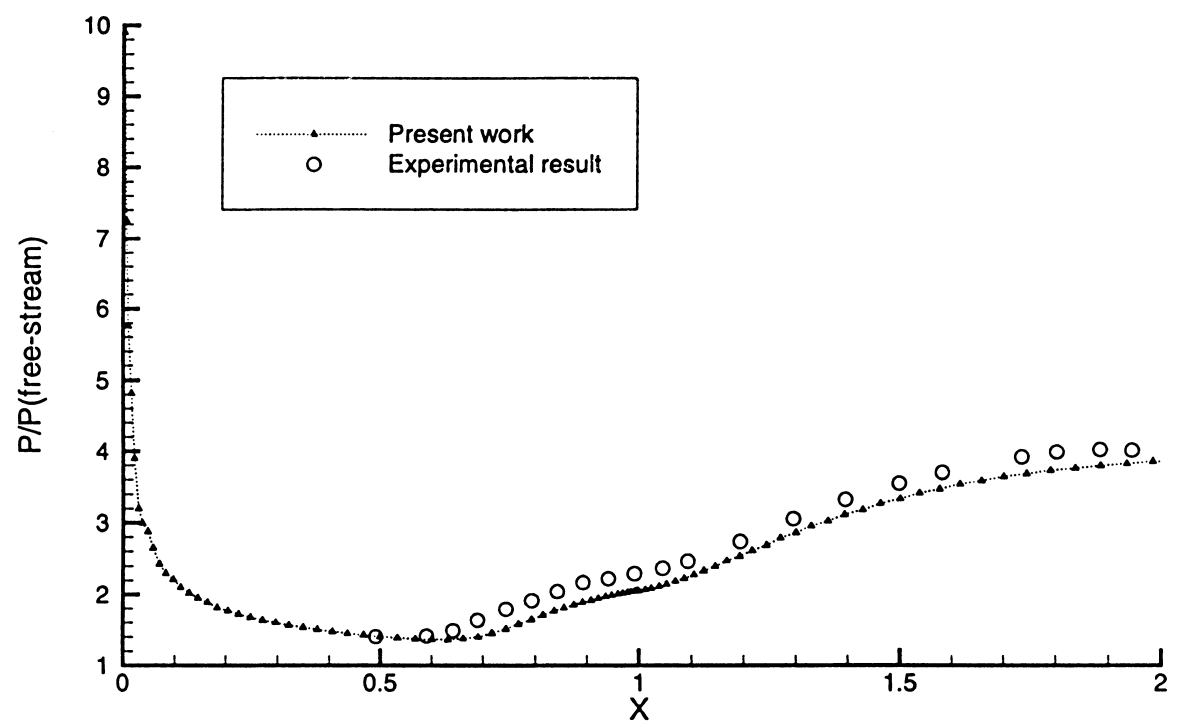

Fig. 22. Normalized pressure distribution along the plate. 
The solution of this problem using the quadratic element is shown in Fig. 21. A shock starts at the leading edge of the plate and propagates through the whole domain. At the corner, a compression shock is developed due to the change of angle of the wall, and a back flow is observed.

Fig. 22 shows the calculated pressure distribution normalized by the free-stream pressure along the plate as well as experimental data [28]. Reasonable agreement is observed.

\section{Conclusions}

Our objective in this work was to develop a versatile and computationally effective parabolic quadrilateral finite element for the solution of compressible flows. We have presented in this paper an element formulation and the results obtained in the solution of various flow problems, in which the Mach number ranged from about 0.0005 to 6 . The numerical results indicate that the element can be used to give reasonably good solutions and is applicable to a wide range of problems. For the problems considered, the solution results using the quadratic element in coarse meshes are comparable to the results produced using other methods with very fine meshes. Of course, further numerical studies and a mathematical analysis of the proposed finite element scheme, especially of the shock capturing term, should be pursued.

When considering very low Mach number solutions, the element formulation should be stable and optimal as has been achieved for elements applicable to incompressible flows [1]. For very low Mach number problems, the element proposed in this paper may suffer from the difficulties that are encountered when the inf-sup condition is not satisfied by a discretization for incompressible flows. Therefore, the formulation still needs to be extended to be more effective for the solution of very low Mach number flow problems.

While the results using the element appear promising, we have not yet considered in our study the details of numerical effectiveness of the element, in particular, the solution of the governing finite element equations. To obtain the solutions of the problems considered in this paper, we used a successive substitution and relaxation method which resulted into slow convergence. An iterative solver specific for the quadratic element should be developed to obtain faster convergence in the solution of the governing equations. We leave these topics of numerical effectiveness for further research.

\section{Acknowledgements}

We would like to thank F. Brezzi, University of Pavia, and D. Chapelle, INRIA, for their comments regarding the paper.

\section{Appendix A. The upwind term}

The objective in this appendix is to give some thoughts regarding the effect of the upwinding in Eq. (10) by considering a simple scalar convective-diffusive problem.

Consider the following one-dimensional problem

$$
\begin{aligned}
& v^{*} \theta_{, x}-\alpha^{*} \theta_{, x x}=q \text { in } 0<x<1, \\
& \theta(0)=\theta(1)=0,
\end{aligned}
$$

where $\theta, \alpha^{*}, q, v^{*}$, are temperature, diffusivity, heat generation and constant velocity. The variables $v^{*}, \alpha^{*}$, and $q$ are given for the problem and we want to solve for $\theta$. We introduce the spaces

$$
V=\left\{v\left|v \in L^{2}(\mathrm{Vol}) ; \frac{\partial v}{\partial x} \in L^{2}(\mathrm{Vol}) ; v\right|_{S_{u}}=0\right\},
$$




$$
\begin{aligned}
& \tilde{V}=\left\{v\left|v \in L^{2}(\mathrm{Vol}) ; \frac{\partial v}{\partial x} \in L^{2}(\mathrm{Vol}) ; \frac{\partial^{2} v}{\partial x^{2}} \in L^{2}\left(\mathrm{Vol}^{(m)}\right) ; v\right|_{S_{u}}=0\right\}, \\
& V_{h}=\left\{v_{h}\left|v_{h} \in L^{2}(\mathrm{Vol}) ; \frac{\partial v_{h}}{\partial x} \in L^{2}(\mathrm{Vol}) ; v_{h} \in Q_{2}\left(\mathrm{Vol}^{(m)}\right) ; v_{h}\right|_{S_{u}}=0\right\},
\end{aligned}
$$

where $Q_{2}\left(\mathrm{Vol}^{(m)}\right)$ denotes the quadratic function of the reference element $m$. $\mathrm{Vol}^{(m)}$ is characterized by the element length $h$.

Introducing the variational formulation for the problem (A.1), the solution $\theta \in V$ is obtained from the following equation

$$
\int_{\mathrm{Vol}}\left(w v^{*} \theta_{, x}+w_{, x} \alpha^{*} \theta_{, x}\right) \mathrm{dVol}=\int_{\mathrm{Vol}} w q \mathrm{dVol} \quad \forall w \in V .
$$

The finite element solution $\theta_{h} \in V_{h}$ of the problem (A.1) is obtained by solving the following equation

$$
\int_{\mathrm{Vol}}\left(w_{h} v^{*} \theta_{h, x}+w_{h, x} \alpha^{*} \theta_{h, x}\right) \mathrm{dVol}=\int_{\mathrm{Vol}} w_{h} q \mathrm{dVol} \quad \forall w_{h} \in V_{h}
$$

As is well-known, the solution shows oscillations as the Peclet number of the problem increases. To stabilize the solution, in our scheme, we add the high-order derivative artificial diffusion term

$$
\sum_{m} \int_{\mathrm{Vol}^{(m)}} w_{h, x x} \alpha^{t} \theta_{h, x x} \mathrm{dVol}^{(m)}
$$

and obtain

$$
\int_{\mathrm{Vol}}\left(w_{h} v^{*} \theta_{h, x}+w_{h, x} \alpha^{*} \theta_{h, x}\right) \mathrm{d} \mathrm{Vol}+\sum_{m} \int_{\mathrm{Vol}^{(m)}} w_{h, x x} \alpha^{t} \theta_{h, x x} \mathrm{dVol}^{(m)}=\int_{\mathrm{Vol}} w_{h} q \mathrm{dVol} \quad \forall w_{h} \in V_{h},
$$

where $\alpha^{t}$ is the artificial diffusion, $\alpha^{t}=o\left(\left|v^{*}\right| h^{3}\right)$, and $h$ is the element length. Note that the upwind term is applied on the element level since the second derivatives $w_{h, x x}$ and $\theta_{h, x x}$ cannot be integrated across the element boundaries.

Adding the high-order derivative artificial diffusion term modifies the original problem considered. Let us consider the consistency of the modified problem with respect to the original problem. The modified problem is to find $\tilde{\theta} \in \tilde{V}$ satisfying

$$
\int_{\mathrm{Vol}}\left(\tilde{w} v^{*} \tilde{\theta}_{, x}+\tilde{w}_{, x} \alpha^{*} \tilde{\theta}_{, x}\right) \mathrm{d} \operatorname{Vol}+\sum_{m} \int_{\operatorname{Vol}^{(m)}} \tilde{w}_{, x x} \alpha^{t} \tilde{\theta}_{, x x} \mathrm{dVol}^{(m)}=\int_{\mathrm{Vol}} \tilde{w} q \mathrm{dVol} \quad \forall \tilde{w} \in \tilde{V}
$$

Assuming a continuous dependence of the solution on the parameter $\alpha^{t}$, consistency of the modified problem with respect to the original problem in Eq. (A.2) follows because the upwind term vanishes as $h \rightarrow 0$ and also the extra constraint in the space definition $(\tilde{V})$ disappears as $h \rightarrow 0$. So, we have that $\tilde{\theta} \rightarrow \theta$ as $h \rightarrow 0$. The consistency of the modified finite element problem in Eq. (A.5) with respect to the original finite element problem in Eq. (A.3) also follows.

To prove that the upwind term stabilizes the solution, we establish an error bound. Consider the consistency condition of the modified problem,

$$
\int_{\mathrm{Vol}}\left\{w_{h} v^{*}\left(\tilde{\theta}-\theta_{h}\right)_{, x}+w_{h, x} \alpha^{*}\left(\tilde{\theta}-\theta_{h}\right)_{, x}\right\} \mathrm{dVol}+\sum_{m} \int_{\mathrm{Vol}^{(m)}} w_{h, x x} \alpha^{t}\left(\tilde{\theta}-\theta_{h}\right)_{, x x} \mathrm{dVol}^{(m)}=0 \quad \forall w_{h} \in V_{h},
$$

where $\tilde{\theta}$ is the exact solution of the modified problem. 
However, also for any $w_{h} \in V_{h}$, we have

$$
\int_{\mathrm{Vol}}\left(w_{h} v^{*} w_{h, x}+w_{h, x} \alpha^{*} w_{h, x}\right) \mathrm{dVol}+\sum_{m} \int_{\mathrm{Vol}^{(m)}} w_{h, x x} \alpha^{t} w_{h, x x} \mathrm{dVol}^{(m)}=\alpha^{*}\left|w_{h}\right|_{1}^{2}+\alpha^{t}\left|w_{h}\right|_{2}^{2} .
$$

Using $w_{h}=\theta_{h}-v_{h}, v_{h} \in V_{h}$, we have

$$
\begin{aligned}
\alpha^{*}\left|\theta_{h}-v_{h}\right|_{1}^{2}+\alpha^{t}\left|\theta_{h}-v_{h}\right|_{2}^{2}= & \int_{\mathrm{Vol}}\left\{\left(\theta_{h}-v_{h}\right) v^{*}\left(\theta_{h}-v_{h}\right)_{, x}+\left(\theta_{h}-v_{h}\right)_{, x} \alpha^{*}\left(\theta_{h}-v_{h}\right)_{, x}\right\} \mathrm{dVol} \\
& +\sum_{m} \int_{\mathrm{Vol}^{(m)}}\left(\theta_{h}-v_{h}\right)_{, x x} \alpha^{t}\left(\theta_{h}-v_{h}\right)_{, x x} \mathrm{dVol}^{(m)} \\
= & \int_{\mathrm{Vol}}\left\{-\left(\theta_{h}-v_{h}\right)_{, x} v^{*}\left(\tilde{\theta}-v_{h}\right)+\left(\theta_{h}-v_{h}\right)_{, x} \alpha^{*}\left(\tilde{\theta}-v_{h}\right)_{, x}\right\} \mathrm{dVol} \\
& +\sum_{m} \int_{\mathrm{Vol}^{(m)}}\left(\theta_{h}-v_{h}\right)_{, x x} \alpha^{t}\left(\tilde{\theta}-v_{h}\right)_{, x x} \mathrm{dVol}^{(m)},
\end{aligned}
$$

where we have used Eq. (A.7) and integration by parts on the convective term.

If we choose $v_{h}$ to satisfy the following constraint equation over each element $m$,

$$
\int_{\mathrm{Vol}^{(m)}}\left(\tilde{\theta}-v_{h}\right) \mathrm{dVol}^{(m)}=0
$$

we have

$$
\begin{aligned}
\int_{\mathrm{Vol}}-\left(\theta_{h}-v_{h}\right)_{, x} v^{*}\left(\tilde{\theta}-v_{h}\right) \mathrm{dVol} & =-\int_{\mathrm{Vol}}\left\{\left(\theta_{h}-v_{h}\right)_{, x}-\operatorname{mean}\left(\left(\theta_{h}-v_{h}\right)_{, x}\right)\right\} v^{*}\left(\tilde{\theta}-v_{h}\right) \mathrm{dVol} \\
& \leqslant\left|v^{*}\right|\left\|\left(\theta_{h}-v_{h}\right)_{, x}-\operatorname{mean}\left(\left(\theta_{h}-v_{h}\right)_{, x}\right)\right\|_{L^{2}}\left\|\tilde{\theta}-v_{h}\right\|_{L^{2}} .
\end{aligned}
$$

Also, we have the following inequality

$$
\begin{aligned}
\left\|\left(\theta_{h}-v_{h}\right)_{, x}-\operatorname{mean}\left(\left(\theta_{h}-v_{h}\right)_{, x}\right)\right\|_{L^{2}} & \leqslant c h\left|\left(\theta_{h}-v_{h}\right)_{, x}\right|_{1} \\
& =c h\left|\theta_{h}-v_{h}\right|_{2},
\end{aligned}
$$

where $c$ is a constant independent of $h$. Hence, Eq. (A.9) becomes

$$
\begin{aligned}
\alpha^{*} \mid \theta_{h} & -\left.v_{h}\right|_{1} ^{2}+\alpha^{t}\left|\theta_{h}-v_{h}\right|_{2}^{2} \\
& \leqslant c h\left|v^{*}\right|\left|\theta_{h}-v_{h}\right|_{2}|| \tilde{\theta}-v_{h} \|_{L^{2}}+\alpha^{*}\left|\theta_{h}-v_{h}\right|_{1}\left|\tilde{\theta}-v_{h}\right|_{1}+\alpha^{t}\left|\theta_{h}-v_{h}\right|_{2}\left|\tilde{\theta}-v_{h}\right|_{2} \\
& \leqslant\left[\alpha^{*}\left|\theta_{h}-v_{h}\right|_{1}^{2}+\alpha^{t}\left|\theta_{h}-v_{h}\right|_{2}^{2}\right]^{\frac{1}{2}}\left[\frac{c^{\prime} h^{2}\left|v^{*}\right|^{2}}{\alpha^{t}}\left\|\tilde{\theta}-v_{h}\right\|_{L^{2}}^{2}+\alpha^{*}\left|\tilde{\theta}-v_{h}\right|_{1}^{2}+2 \alpha^{t}\left|\tilde{\theta}-v_{h}\right|_{2}^{2}\right]^{\frac{1}{2}},
\end{aligned}
$$

where $c^{\prime}$ is also a constant independent of $h$. Rewriting the above equation, we have

$$
\alpha^{*}\left|\theta_{h}-v_{h}\right|_{1}^{2}+\alpha^{t}\left|\theta_{h}-v_{h}\right|_{2}^{2} \leqslant \frac{c^{\prime} h^{2}\left|v^{*}\right|^{2}}{\alpha^{t}}\left\|\tilde{\theta}-v_{h}\right\|_{L^{2}}^{2}+\alpha^{*}\left|\tilde{\theta}-v_{h}\right|_{1}^{2}+2 \alpha^{t}\left|\tilde{\theta}-v_{h}\right|_{2}^{2} .
$$

So that by the triangle inequality, for all $v_{h} \in V_{h}$ satisfying the constraint equation (Eq. (A.10))

$$
\begin{aligned}
\alpha^{*}\left|\tilde{\theta}-\theta_{h}\right|_{1}^{2}+\alpha^{t}\left|\tilde{\theta}-\theta_{h}\right|_{2}^{2} & =\alpha^{*}\left|\tilde{\theta}-v_{h}+v_{h}-\theta_{h}\right|_{1}^{2}+\alpha^{t}\left|\tilde{\theta}-v_{h}+v_{h}-\theta_{h}\right|_{2}^{2} \\
& \leqslant \alpha^{*}\left(\left|\tilde{\theta}-v_{h}\right|_{1}^{2}+\left|v_{h}-\theta_{h}\right|_{1}^{2}\right)+\alpha^{t}\left(\left|\tilde{\theta}-v_{h}\right|_{2}^{2}+\left|v_{h}-\theta_{h}\right|_{2}^{2}\right) \\
& \leqslant \frac{c^{\prime} h^{2}\left|v^{*}\right|^{2}}{\alpha^{t}}\left\|\tilde{\theta}-v_{h}\right\|_{L^{2}}^{2}+2 \alpha^{*}\left|\tilde{\theta}-v_{h}\right|_{1}^{2}+3 \alpha^{t}\left|\tilde{\theta}-v_{h}\right|_{2}^{2} .
\end{aligned}
$$

Considering the case $\alpha^{*}>0$, this relation shows that convergence is reached in the finite element solution. The relation also shows that the choice of $\alpha^{t}=\mathrm{o}\left(h^{3}\left|v^{*}\right|\right)$ is a reasonable one. Of course, further analysis is necessary in order to identify the more detailed behavior of the solution scheme. 


\section{References}

[1] K.J. Bathe, Finite element procedures, Prentice-Hall, Englewood Cliffs, NJ, 1996.

[2] K.J. Bathe, H. Zhang, X. Zhang, Some advances in the analysis of fluid flows, Comput. Structures 64 (1997) 909-930.

[3] P.R.M. Lyra, K. Morgan, J. Peraire, J. Peiro, TVD algorithms for the solution of the compressible Euler equations on unstructured meshes, Int. J. Numer. Methods Fluids 19 (1994) 827-847.

[4] M. Fortin, H. Manouzi, A. Soulaimani, On finite element approximation and stabilization methods for compressible viscous flows, Int. J. Numer. Methods Fluids 17 (1993) 477-499.

[5] A. Soulaimani, M. Fortin, Finite element solution of compressible viscous flows using conservative variables, Comput. Methods Appl. Mech. Engrg. 118 (1994) 319-350.

[6] R.C. Almeida, A.C. Galeao, An adaptive Petrov-Galerkin formulation for the compressible Euler and Navier-Stokes equations, Comput. Methods Appl. Mech. Engrg. 129 (1996) 157-176.

[7] H. Luo, J.D. Baum, R. Löhner, J. Cabello, Implicit schemes and boundary conditions for compressible flows on unstructured grids, AIAA paper 94-0816, 1994.

[8] G.J.L. Beau, S.E. Ray, S.K. Aliabadi, T.E. Tezduyar, SUPG finite element computation of compressible flows with the entropy and conservation variables formulations, Comput. Methods Appl. Mech. Engrg. 104 (1993) 397-422.

[9] R.A. Shapiro, Adaptive finite element solution algorithm for the Euler equations, Notes on numerical fluid mechanics, Vol. 32, Vieweg, Braunschweig, 1991.

[10] S.R. Chakravarthy, Euler equations - Implicit schemes and boundary conditions, AIAA J. 21 (1983) 699-706.

[11] L. Demkowicz, J.T. Oden, W. Rachowicz, O. Hardy, An $h-p$ Taylor-Galerkin finite element method for compressible Euler equations, Comput. Methods Appl. Mech. Engrg. 88 (1991) 363-396.

[12] L. Demkowicz, J.T. Oden, W. Rachowicz, O. Hardy, Toward a universal $h-p$ adaptive finite element strategy, part 1. constrained approximation and data structure, Comput. Methods Appl. Mech. Engrg. 77 (1989) 79-112.

[13] J.T. Oden, L. Demkowicz, W. Rachowicz, L. Westermann, A posteriori error analysis in finite elements: The element residual method for symmetrizable problems with applications to compressible Euler and Navier-Stokes equations, Comput. Methods Appl. Mech. Engrg. 82 (1990) 183-203.

[14] W.W. Tworzydlo, J.T. Oden, E.A. Thornton, Adaptive implicit/explicit finite element method for compressible viscous flows, Comput. Methods Appl. Mech. Engrg. 95 (1992) 397-440.

[15] A.N. Brooks, T.J.R. Hughes, Streamline upwind/Petrov-Galerkin formulations for convection dominated flows with particular emphasis on the incompressible Navier-Stokes equations, Comput. Methods Appl. Mech. Engrg. 32 (1982) 199-259.

[16] T.J.R. Hughes, M. Mallet, A new finite element formulation for computational fluid dynamics: III. The generalized streamline operator for multidimensional advective-diffusive systems, Comput. Methods Appl. Mech. Engrg. 58 (1986) $305-328$.

[17] J. Donea, A generalized Galerkin method for steady convection-diffusion problems with application to quadratic shape function elements, Comput. Methods Appl. Mech. Engrg. 48 (1985) 25-43.

[18] R. Codina, E. Oñate, M. Cervera, The intrinsic time for the streamline upwind/Petrov-Galerkin formulation using quadratic elements, Comput. Methods Appl. Mech. Engrg. 94 (1992) 239-262.

[19] D.L. Hill, E.A. Baskharone, A monotone streamline upwind method for quadratic finite elements, Int. J. Numer. Methods Fluids 17 (1993) 463-475.

[20] B.M. DeBlois, Quadratic streamline upwinding for finite element method solution to 2-D convective transport problems, Comput. Methods Appl. Mech. Engrg. 134 (1996) 107-115.

[21] B.P. Leonard, The ULTIMATE conservative difference scheme applied to unsteady one-dimensional advection, Comput. Methods Appl. Mech. Engrg. 88 (1991) 17-74.

[22] T.J.R. Hughes, M. Mallet, A new finite element formulation for computational fluid dynamics: IV. A discontinuity-capturing operator for multidimensional advective-diffusive systems, Comput. Methods Appl. Mech. Engrg. 58 (1986) 329-336.

[23] C. Hirsch, Numerical Computation of Internal and External Flows, Vol. 2, Wiley, New York, 1990.

[24] X. Wang, K.J. Bathe, Displacement/pressure based mixed finite element formulations for acoustic fluid-structure interaction problems, Int. J. Numer. Methods Engrg. 40 (1997) 2001-2017.

[25] ADINA R\&D, Inc., ADINA-F user manuals, Watertown, Massachusetts, 1996.

[26] F. Shakib, T.J.R. Hughes, Z. Johan, A new finite element formulation for computational fluid dynamics: X. The compressible Euler and Navier-Stokes equations, Comput. Methods Appl. Mech. Engrg. 89 (1991) 141-219.

[27] A.F. Mills, Heat Transfer, Irwin, Homewood, IL, 1992.

[28] J.E. Lewis, T. Kubota, L. Lees, Experimental investigation of supersonic laminar, two-dimensional boundary-layer separation on a compression corner with and without cooling, AIAA J. 6 (1968) 7-14. 